\title{
REVISIÓN TÉCNICA DEL ESTUDIO DE SOSTENIBILIDAD DEL RÉGIMEN DE INVALIDEZ, VEJEZ Y MUERTE COSTARRICENSE
}

\author{
Juan J. Víquez ${ }^{1}$ \\ Edgar A. Robles ${ }^{2}$
}

Recibido: 01/11/2018

Aprobado: 07/06/2019

\begin{abstract}
RESUMEN
En este artículo se analizan algunas de las debilidades encontradas en el Estudio Actuarial del Régimen de Invalidez, Vejez y Muerte (IVM), realizado por el equipo de la Escuela de Matemática de la Universidad de Costa Rica (UCR). Se efectúa un análisis sobre la población económicamente activa (PEA) utilizada por ellos, $y$ se demuestra que esta base poblacional se encuentra inicialmente subestimada, al tiempo en que se realiza una revisión del modelo, especificando algunas incoherencias demográficas que se derivan de la implementación errónea del diagrama de Lexis asociado. Posteriormente, se analizan los supuestos implícitos del modelo, los cuales afectan directamente los resultados del estudio, $y$ se agregan posibles mejoras al modelo que devienen en un aumento de la exactitud y certeza de los resultados del informe actuarial. Como conclusión general, se estima que los resultados del estudio del IVM realizado por la UCR presentan sesgos que corresponden a un escenario pesimista.
\end{abstract}

PALABRAS CLAVE: ACTUARIAL, PENSIONES, BACK-TESTING.

CLASIFICACIÓN JEL: J110; H000; J180

ABSTRACT

This article analyses some of the weaknesses found in the actuarial study of the biggest national pension fund named "Régimen de Invalidez, Vejez y Muerte" (IVM), performed by a team from the Mathematics School at the Universidad de Costa Rica (UCR). The paper conducts an analysis on the economically active population used by them, showing that this demographic data is initially underestimated. The paper also reviews the population generation model, stating the demographic incoherencies derived from a mistaken implementation of the associated Lexis diagram. Afterwards, the paper analyzes the assumptions implicit in the model, specifying how they affect the model results, and finally it gives some solutions to improve the accuracy of the forecasting. As a general conclusion, it is estimated that the results of the IVM study conducted by the UCR are biased and correspond to a pessimistic scenario.

KEY WORDS: ACTUARIAL, PENSIONS, BACK-TESTING.

JEL CLASIFICATION: J110; H000; J180

1 Universidad de Costa Rica, Escuela de Matemática; Código postal 11501-2060; San José, Costa Rica; viquezejin@gmail.com 


\section{INTRODUCCIÓN}

En los últimos 15 años, la sostenibilidad del Régimen de Invalidez, Vejez y Muerte (IVM), administrado por la Caja Costarricense de Seguro Social (CCSS), se ha puesto en duda a través de varios estudios actuariales, tanto nacionales como externos. Entre estos estudios se encuentran:

- Dirección Actuarial y Económica de la CCSS (2015, p. 39-40).

- Organización Internacional del Trabajo (OIT, 2010, p. 21), a solicitud de la CCSS.

- Nathal Actuarios y Consultores (Nathal, 2008, p. 36), por contratación directa de la Superintendencia de Pensiones (SUPEN).

- Dr. Eduardo Melinsky (Melinsky, 2015, p. 19), por licitación conjunta CCSS-SUPEN.

Todos los informes, independientemente de la metodología y datos utilizados, tienen una conclusión en común: el IVM presenta un riesgo de desfinanciamiento, desde el punto de vista de sostenibilidad actuarial. El proceso que conduciría a este desenlace, según dichos informes, es el siguiente: en un primer momento, el IVM comenzó a hacer uso de los intereses de la reserva para enfrentar sus obligaciones ${ }^{3}$; en una segunda etapa, en un futuro, se verá obligado a utilizar la reserva; y finalmente, dicha reserva se agotará.

Es válido preguntarse ¿por qué es necesario un estudio actuarial de la Escuela de Matemática de la Universidad de Costa Rica (UCR), si ya todos los estudios anteriores comparten la misma conclusión? La razón principal es que las fechas que cada estudio actuarial arroja para los dos últimos momentos críticos distan mucho entre sí. El informe IVM-UCR de la Escuela de Matemática (Arias, Barboza \& Ramírez, 2016) no debe responder si el IVM es actuarialmente solvente, pues ya se sabe la respuesta a esta pregunta, sino que debería dilucidar la siguiente interrogante:

¿En cuáles años se espera que sucedan los dos puntos de quiebre: uso de la reserva e imposibilidad de pagar todas las pensiones?

La respuesta a esta pregunta es de vital para la toma de decisiones, especialmente si se cuantifica la magnitud del déficit en el momento de quiebra. Existen muchas estrategias que se pueden implementar para traer sostenibilidad al fondo, unas que toman más tiempo para su entrada en funcionamiento, $y$ otras a más corto plazo, pero con mayor impacto, las cuales podrían tener efectos "secundarios" indeseables dentro del fondo.

Por ejemplo, si un estudio actuarial realizado en el 2015 indica que para el 2022 ya no existen reservas y el fondo "quiebra", entonces las medidas a implementar deben ser de choque, incluyendo cargas al Estado para que rescate dicho régimen; pero si, por el contrario, el estudio muestra que el momento de insostenibilidad sucede en el año 2040, entonces se pueden aplicar medidas graduales, que requieran varios años para su implementación, pero cuyo impacto sería el requerido.

En virtud de la importancia que tienen los resultados obtenidos en el estudio actuarial de la Escuela de Matemática de la UCR, cualquier debilidad técnica debe ser analizada detenidamente, intentando corregirla, o en su defecto, cuantificar su impacto dentro de la sostenibilidad del IVM. Es por ello, que en este artículo se realiza una revisión exhaustiva del modelo utilizado por los encargados de realizar el estudio actuarial IVM-UCR (Arias et al., 2016), así como de sus supuestos, datos empleados, proyecciones generadas, resultados y conclusiones. A lo largo de este proceso, se lograron detectar inexactitudes en la generación poblacional, el modelo implementado, los datos que alimentaron al modelo, el ajuste de los parámetros (tales como las densidades de cotización), y demás falencias, que son presentadas en este artículo académico.

3 De acuerdo con la OIT (2010) y la Dirección Actuarial y Económica de la CCSS (2015), este momento se presentó en el año 2011. Ver también el cuadro 105 de Arias, Barboza y Ramírez (2016). 
Se enfatiza que varias de estas observaciones les fueron expuestas al equipo actuarial de la Escuela de Matemática, pocos días después de que ellos entregaran el estudio actuarial en diciembre de 2016. En una reunión tripartita entre las autoridades de la CCSS, la SUPEN, y el equipo de la Escuela de Matemática de la UCR, se acordó que, ante las dudas presentadas, Arias, Ramírez y Barboza realizarían pruebas de Back-Testing, para validar el modelo utilizado y confirmar así la idoneidad de la modelación.

Sin embargo, en junio de 2017, se indicó que dichas pruebas no fueron realizadas, lo cual motivó la confección de este artículo académico, esperando iniciar así un debate académico, intelectual, técnico y objetivo, donde se analicen los argumentos aquí esgrimidos y se arroje luz a la necesidad de revisar las conclusiones provenientes de un modelo que requiere de mayor robustez, producto de su metodología.

Este artículo se encuentra dividido de la siguiente manera. En la segunda sección, se describe la metodología utilizada en el análisis, donde se presenta el modelo de la OIT, el modificado por la UCR y las posibles correcciones a dicha adaptación. También se explican las distintas poblaciones construidas para contrastar los resultados del modelo de la UCR, tales como la PEA que se debió utilizar, o la población inflada que no puede ser superada por los pertenecientes al IVM, entre otros. En la tercera sección, se trabajan tres tipos de mejoras que se le pueden incorporar a un nuevo estudio, correspondientes a: datos empleados, modelo utilizado, supuestos e implementación. Se realiza el análisis y una estimación del impacto de estas mejoras dentro de los resultados del estudio del IVM. En la sección cuatro, se presentan las conclusiones del análisis, en las cuales, a partir de lo mostrado en las secciones anteriores, se considera que los resultados del informe IVM-UCR (Arias et al., 2016), realizado por la Escuela de Matemáticas de la UCR, no son fiables pues contienen deficiencias técnicas.

\section{METODOLOGÍA}

En este análisis se utilizan los documentos de Excel utilizados por el equipo de la Escuela de Matemática, donde se presentan los datos suministrados por la CCSS sobre PEA, cotizantes, afiliados, pensionados, entre otros; así como los datos probabilísticos obtenidos del histórico entregado a ellos por parte de la CCSS, tales como las densidades de cotización o las tablas biométricas. A lo largo de este artículo, se analiza la coherencia demográfica y la modelación matemática, para estimar el impacto de las deficiencias encontradas en el estudio actuarial del IVM realizado por la UCR.

Antes que todo, es importante aclarar algunos conceptos que serán de vital importancia para comprender las variables involucradas en el presente análisis.

1. Población oficial: se considera la población generada por el Instituto Nacional de Estadística y Censos junto con el Centro Centroamericano de Población (INEC-CCP, 2013) como la "población oficial".

2. Población alternativa: en el estudio IVM-UCR (Arias et al., 2016), se emplean los datos del Centro Latinoamericano y Caribeño de Demografía (CELADE) para comparar los radios de soporte calculados por ellos versus los poblacionales (Arias et al., 2016, p. 42), de modo que en ocasiones se utilizará esta población, en conjunto con la población oficial, para evidenciar algunas contradicciones demográficas. La diferencia entre la población oficial y la población alternativa radica en que la primera utiliza las bases de datos del INEC y del CCP para construir las proyecciones, mientras que la segunda utiliza estadísticas más generales para realizarlas, de ahí que la población oficial sea más fiable.

3. Afiliados y cotizantes: en este artículo se utilizan las definiciones de "afiliados" y "cotizantes” empleadas en el estudio IVM-UCR (Arias et al., 2016, p. 55). Más específicamente, un afiliado es todo aquel individuo que haya cotizado al menos una vez. Por su parte, un cotizante es un afiliado que está actualmente cotizando. 
A continuación, se presentan los fundamentos teóricos, junto con las construcciones probabilísticas y demográficas, requeridas para la confección del análisis que sigue a esta sección. Se inicia con la metodología empleada por la CCSS para proyectar la PEA, para posteriormente construir una PEA con los datos poblacionales del Centro Centroamericano de Población (CCP) y el Instituto Nacional de Estadística y Censos (INEC) (2013).

\section{Población económicamente activa (PEA-CCSS)}

El estudio IVM-UCR (Arias et al., 2016) utilizó una proyección de PEA elaborada en 2014 por la Dirección Actuarial y Económica (2014). Esta proyección sigue una metodología basada en proyectar las tasas futuras de actividad económica por sexo y edad y aplicar esas tasas a las proyecciones oficiales de población del país. No obstante, el citado estudio incurrió en dos sesgos durante la aplicación de esta metodología, los cuales se citan a continuación:

- Tasas de participación subestimadas: se asume una caída futura de las tasas de participación de los hombres en las edades de máxima productividad (25 a 49 años de edad), pasando de un $98 \%$ de participación en 2015, a 78\% en 2100. Esta caída de 20 puntos porcentuales es difícil de justificar. Por ejemplo, la CEPAL en su proyección de PEA para Costa Rica (CELADE, 2016) supone unas tasas de participación de los hombres en 2050 (último año de su proyección) de 95\% a los 25-29 años de edad, y de 98\% a 100\% entre los 30 y 49 años. Aunque para las mujeres la proyección de la CCSS asume razonablemente una convergencia hacia los niveles de participación de los hombres (las tasas de las mujeres llegarán a ser 96\% de las tasas de los hombres en 2100), las tasas de las mujeres asumidas en la proyección son bajas debido a que las tasas de los hombres también los son.

- Proyección de Población Equivocada a Partir de 2050: entre 2015 y 2050 se usaron correctamente las proyecciones de población oficiales (INEC-CCP, 2013), pero de 2050 a 2100 la Dirección Actuarial y Económica de la CCSS supuso un crecimiento "geométrico" o "Malthusiano" de la población (CCSS, 2014, p. 2-6), sin que ese crecimiento pueda ser explicado técnicamente. Las proyecciones del INEC-CCP (2013) indican que entre 2050 y 2100 la población en edad de trabajar probablemente no crecerá y, al contrario, disminuirá de 3,4 millones en 2015 a 3,2 millones en 2100.

Para aquilatar la magnitud del problema generado por estos dos sesgos en la proyección de la PEA-CCSS, se efectuó una nueva proyección denotada como "PEA-INEC-CCP", utilizando principalmente los siguientes dos supuestos.

- Tasas de participación de los hombres: la participación de los hombres se modificará poco, solo decrecerá ligeramente entre los jóvenes, debido a una mayor retención escolar, y entre los adultos mayores, debido a una mayor cobertura de la seguridad social. En las edades más productivas, las tasas permanecerán constantes y cercanas a 100\%. Nótese que estos supuestos son similares a los de la proyección de PEA de la CEPAL en (CELADE, 2017).

- Tasas de participación de las mujeres: se supone una convergencia hacia niveles de participación de los hombres de manera que en 2100 las tasas de las mujeres serán el 90\% de las tasas de los hombres. Se supone también que este incremento de la participación de las mujeres seguirá una curva logística. 
- Población base: las tasas de participación se combinan con la proyección de población por sexo y edad de INEC-CCP (2013) para obtener la PEA4.

Para completar el análisis, se construyen las "tasas de actividad implícitas", esto es, se utiliza la PEA-CCSS y la población INEC-CCP, filtrada para las edades entre 60-64 años, de modo que la tasa de actividad implícita sería el cociente de la primera entre la segunda, y se obtiene la proporción de personas en ese rango de edad que están en la PEA. Asimismo, se contrastan los "niveles de cobertura implícitos" en las proyecciones del estudio IVM-UCR (Arias et al., 2016), para lo cual se hace uso del cociente entre los cotizantes generados por el modelo y la PEA-INEC-CCP.

Se debe recordar que la PEA-CCSS utilizada en el informe IVM-UCR (Arias et al., 2016), consiste de aquellas personas mayores a 15 años que están laborando o se encuentran buscando trabajo (desempleados). Sin embargo, existen personas que no forman parte de ese subgrupo, ya sea porque están estudiando o simplemente no laboran (ni están buscando trabajar). Por esta razón, la PEACCSS no podría exceder el total de personas (para cada rango de edades).

\section{Modelo Matemático-Actuarial}

En esta subsección se aborda el modelo empleado y las correcciones de lo que se considera son debilidades en la modelación e implementación. Antes de iniciar, se debe aclarar que se considera acertada la utilización de la base completa de afiliados dentro del estudio, en lugar de sólo trabajar con los cotizantes del 2015.

Se inicia presentando, a grandes rasgos, el modelo de la Organización Internacional del Trabajo (Subramaniam, 1999), el cual sirvió de base para el modelo de la UCR. Se explican las principales adaptaciones que se realizaron al modelo, por parte del equipo de la Escuela de Matemática (Arias et al., 2016), para finalizar explicando las correcciones que se deben hacer para que dicha modificación al modelo sea probabilísticamente correcta. Se abarcan los insumos del modelo, como la "Densidad de Cotización", así como algunos productos del mismo, tales como "nuevas generaciones", "cotizantes" y "afiliados".

\section{Modelo OIT}

En OIT (Subramaniam, 1999, p. 70) se presenta un modelo de generación de población cotizante y pensionada, pero para un escenario donde no existen afiliados no-cotizantes, es decir, si es afiliado entonces cotiza. Para ello, el modelo trabaja con las siguientes variables:

- $\operatorname{Cot}_{x, n}$ : Proyección de la población cotizante con edad "x", para el año "n" (determinada exógenamente).

- $\operatorname{Cot}_{x, t, n}$ : Población cotizante con edad "x" y "t" años de cotizar, para el año "n".

- $\quad N G_{x, n}$ : Nuevas generaciones, personas que nunca habían estado en el sistema e ingresan en el año "n" con edad "x".

- $\quad \operatorname{Ret}_{x, t, n}^{j}$ : Población en el estado "j" con edad "x" y "t” años de cotizar, en el año "n".

$\bullet$

- $D_{x, t, n}^{\text {Modelo }}$ : Densidad de cotizaciones que realiza en un año una persona de edad "x" con " $\mathrm{t}$ " años de cotizar, para el n-ésimo año.

4 Estas proyecciones están disponibles hasta el año 2100 en el sitio web: https://censos.ccp.ucr.ac.cr/index.php/ censos_c?censo=crproyna. 
- $\quad p_{x}^{\tau}$ : Probabilidad de sobrevivir a los decrementos de invalidez, retiro, y muerte, para una persona de edad "x".

- $\quad p_{x}^{j}$ : Probabilidad de sobrevivir a los demás decrementos, para una persona de edad "x" en el estado "j".

- $\quad q_{x}^{i j}$ : Probabilidad de pasar del estado "i” al estado "j", para una persona de edad "x".

- $\mathrm{E}\left[\operatorname{Cot} S_{x, t, n}\right]$ : Valor esperado de cotizantes sobrevivientes para el siguiente año, con edad "x" $y$ "t" años de cotizar.

El modelo definido por la OIT sigue la lógica del diagrama de Lexis (ver Bowers, Gerber, Hickman, Jones y Nesbitt, 1997, p. 585), utilizando el hecho de que como los cotizantes no necesariamente cotizan todos los meses del año, la densidad de cotización sirve para dividir a la población de cotizantes entre aquellos que acumulan las "suficientes" cotizaciones como para acceder al nivel de " $t+1$ " años de cotizar en el siguiente año, y aquellos que no alcanzan las cotizaciones para dicha transición, quienes en contraposición se mantienen con los mismos años de cotización.

Esto es necesario debido a que las cotizaciones se realizan mensualmente, pero las estimaciones son anuales, por lo que, de no utilizar este filtro, a una persona que cotice una vez al año se le atribuirían 12 cotizaciones en cada iteración anual del modelo. De este modo, la cantidad esperada de cotizantes con " $t+1$ " años de cotizar deben ser aquellos cotizantes con " $t$ " años de cotizar y que sobrevivieron, más aquellos que tenían " $t+1$ " años de cotizar pero que en ese año no cotizaron, aunque sí sobrevivieron, esto es,

$$
\mathrm{E}\left[\operatorname{Cot}_{x+1, t+1, n+1}\right]=\overbrace{\underbrace{\operatorname{Cot}_{x, t, n} \cdot D_{x, t, n}^{\text {Modelo }} \cdot p_{x}^{\tau}}_{1 \text { año más de cotizaciones }}+\underbrace{\operatorname{Cot}_{x, t+1, n} \cdot\left(1-D_{x, t+1, n}^{\text {Modelo }}\right) \cdot p_{x}^{\tau}}_{\text {No acumulan un año más de cotizaciones }}}^{\text {Todos son cotizantes }}
$$

Las "nuevas generaciones", aquellos que entran al sistema por primera vez, son calculadas como la diferencia entre los cotizantes sobrevivientes del año anterior y los cotizantes "meta". Estos últimos se definen como porcentaje de la PEA (dados los niveles de cobertura acordados), representando la cantidad de cotizantes que deberían existir, esto es,

$$
N G_{x, n}=\frac{\overbrace{\operatorname{Cot}_{x, n}-\sum_{t \geq 0} \mathrm{E}\left[\operatorname{Cot} S_{x, t, n}\right]}^{\text {Asume que entran a mitad de año }}}{p_{x-0,5: 0.5}^{\tau}}
$$

Note que con las nuevas generaciones se acrecientan los cotizantes, provocando que la base sobre la cual se calculan los pensionados esté siendo alimentada por ellos. Tanto los cotizantes esperados como las nuevas generaciones juegan un papel importante en la determinación de las personas pensionadas. 


\section{Modelo UCR (Arias et al., 2016)}

El modelo de la OIT de la subsección anterior, no debería ser aplicado directamente para el Régimen de Pensiones del IVM, debido a que no considera a todos los afiliados, definidos como aquellos que han cotizado al menos una vez en su vida, razón por la cual el equipo de la Escuela de Matemáticas de la UCR se vio en la necesidad de tropicalizarlo.

El modelo adaptado utiliza una nueva variable llamada "Densidad de Cotización", definida como "el valor esperado de la frecuencia de cotización anual futura" (Arias et al., 2016, p. 159), o más específicamente, "es la densidad de cotización promedio del período 2011-2015 para los afiliados vivos y no pensionados con al menos una cuota acreditada" (Arias et al., 2016, p. 242).

Se define lo siguiente:

- $A f_{x, t, n}$ : Población afiliada con edad "x" y "t" años de cotizar, en el año "n".

- $D_{x, t, n}^{U C R}$ : Densidad de cotizaciones que realiza en un año un afiliado de edad "x" con " $\mathrm{t}$ " años de cotizar, para el n-ésimo año.

Para cada género, edad "x" $\mathrm{y}$ "t" años cotizados, la densidad de cotización para el año " $\mathrm{n}$ " $\left(D_{x, t, n}^{U C R}\right)$ se calcularía como la suma de los tiempos cotizados sobre la suma de los tiempos de exposición de los afiliados totales. En términos sencillos, esta densidad de cotización indicaría que para afiliados de edad "x" $y$ con "t" años cotizados, el número de cotizaciones esperadas en este año sería $12 \cdot A f_{x, t} \cdot D_{x, t}^{U C R}$

Considere la siguiente variable:

- $\quad P_{x, t, n}$ : Probabilidad de ser afiliado cotizante (ignorada por la UCR).

Existe una relación directa entre los afiliados cotizantes y los afiliados totales, así como entre la densidad de cotización de afiliados $\left(D_{x, t, n}^{U C R}\right)$ y la de cotizantes $\left(D_{x, t, n}^{\text {Modelo }}\right)$, dada por:

$$
\operatorname{Cot}_{x, t, n}=A f_{x, t, n} \cdot P_{x, t, n} \text { y } D_{x, t, n}^{U C R}=P_{x, t, n} \cdot D_{x, t, n}^{\text {Modelo }}
$$

Note que los afiliados con edad " $\mathrm{x}+1$ ” $\mathrm{y}$ " $\mathrm{t}+1$ " años de cotizar, provienen de tres fuentes:

- Cotizantes fuertes: aquellos afiliados que tenían edad "x", "t" años de cotizar, y sobrevivieron un año más, que además fueron cotizantes ese año y cotizaron "lo suficiente» para pasar a tener " $t+1$ " años de cotizar.

- Cotizantes débiles: aquellos que tenían edad " $x$ ", " $t+1$ " años de cotizar, y sobrevivieron un año más, pero que, a pesar de ser cotizantes no lograron tener el suficiente número de cotizaciones necesarias como para pasar al nivel " $t+2$ ".

- No cotizantes: aquellos que tenían edad "x", " $t+1$ " años de cotizar, y sobrevivieron un año más, pero que no cotizaron ese año.

Si se usa esta relación (3), es posible validar la fórmula empleada por la UCR en el informe IVM-UCR (Arias et al., 2016, p. 243), notando que la cantidad esperada de afiliados sobrevivientes es correcta bajo la siguiente igualdad, 


$$
\begin{aligned}
\mathrm{E}\left[A f S_{x+1, t+1}\right] & =\underbrace{A f_{x, t} \cdot \overbrace{P_{x, t} \cdot D_{x, t}^{\text {Modelo }}}^{=D_{x, t}^{U C R}} \cdot p_{x}^{\tau}}_{\text {Cotizantes fuertes }}+\underbrace{\mathrm{Af}_{\mathrm{x}, t+1} \cdot \overbrace{P_{x, t+1}\left(1-D_{x, t+1}^{\text {Modelo }}\right)}^{=P_{x, t+1}-D_{x, t+1}^{U C R}} \cdot p_{x}^{\tau}}_{\text {Cotizantes débiles }}+\mathrm{Af}_{\mathrm{x}, t+1} \cdot \underbrace{\left(1-P_{x, t+1}\right) \cdot p_{x}^{\tau}}_{\text {No cotizantes }} \\
& =\underbrace{A f_{x, t} \cdot D_{x, t}^{U C R} \cdot p_{x}^{\tau}+A f_{x, t+1} \cdot\left(1-D_{x, t+1}^{U C R}\right) \cdot p_{x}^{\tau}}_{\text {Fórmula usada por la UCR }}
\end{aligned}
$$

En el estudio IVM-UCR (Arias et al., 2016), se utiliza $D_{x, t, n}^{U C R}$ como si fuera la probabilidad de ser cotizante $\left(P_{x, t, n}\right)$, de modo que cuando se estiman a los cotizantes sobrevivientes esperados (Arias et al., 2016, p. 244), con edad " $\mathrm{x}+1$ " $\mathrm{y}$ " $\mathrm{t}+1$ ” años de cotizar, utilizan la siguiente fórmula:

$$
\mathrm{E}\left[\operatorname{Cot} S_{x+1, t+1}\right]=A f_{x, t} \cdot D_{x, t}^{U C R} \cdot p_{x}^{\tau}
$$

Siguiendo con esta lógica, el estudio IVM-UCR (Arias et al., 2016) modifica la fórmula (2) con que se calculan las nuevas generaciones. De acuerdo con el informe IVM-UCR (Arias et al., 2016, p. 243), la distribución de las nuevas generaciones es constante para todos los 100 años de proyección, y es calculada como el promedio de los años 2014 y 2015. Utilizar dos años para estimar parámetros que serán utilizados para generar cien años no es estadísticamente robusto y puede conducir a sesgos. Además, en lugar de calcular las nuevas generaciones para cada edad, se utilizan datos agregados, tanto para la PEA, cotizantes, cotizantes sobrevivientes, y nuevas generaciones.

El algoritmo empleado por el equipo de la UCR (Arias et al., 2016, p. 243) sería:

1. Calcule la cantidad $\mathrm{Cn}$ de cotizantes totales (todas las edades) para el año "n", como el porcentaje de cobertura de la PEA total (todas las edades).

2. Calcule la cantidad CSn de cotizantes sobrevivientes totales (todas las edades) para el año "n"5.

3. Calcule las nuevas generaciones totales para el año " $\mathrm{n}$ " como $\mathrm{NG}_{n}=\mathrm{C}_{n}-\mathrm{CS}_{n}$.

4. Utilice la distribución dngx de las nuevas generaciones totales para obtener las nuevas generaciones de edad " $\mathrm{x}$ ", es decir,

$$
N G_{n}(x)=d n g_{x} \cdot N G_{n}
$$

\section{Mejoras teóricas al modelo UCR}

Hasta ahora no parece presentar ningún problema, sin embargo, existen varios conflictos probabilísticos con la implementación que realizaron los matemáticos de la UCR al no considerar la probabilidad $P_{x, t, n}$. En el estudio IVM-UCR (Arias et al., 2016) la densidad de cotización juega el papel de ambos, de "densidad de cotización" de afiliados, y de "probabilidad de ser cotizante". Aunque inicialmente parecieran ser lo mismo, técnicamente no lo son. Por ejemplo, se podría estimar la den-

5 Como se demuestra en la siguiente subsección, este monto está subestimado, lo que aumenta artificialmente la cantidad de nuevas generaciones. 
sidad de cotización de un afiliado como lo hizo el equipo de la Escuela de Matemática, tratando de responder ¿cuántas cotizaciones hace al año un afiliado estándar? Sin embargo, si se desea estimar la probabilidad de ser un afiliado cotizante, se necesitaría contar el número de afiliados que cotizaron al menos una vez al año, $y$ dividirle el total de afiliados de ese año.

Utilizar la densidad de cotización como una probabilidad de ser cotizante es un error probabilístico que genera problemas en el diagrama de Lexis del modelo. Primero que todo, hay que entender que la única forma de que $D_{x, t, n}^{U C R}=P_{x, t, n}$ es si todos los afiliados cotizantes pagaran sus cotizaciones durante los 12 meses del año (ninguno cotiza menos meses), en cuyo caso las cotizaciones serían una Bernoulli, siendo 0 si es no cotizante y 1 si es cotizante. Sin embargo, este no es el caso que nos ocupa, y se procede a demostrar el error con un ejemplo numérico, en el cual, por sencillez, se ignorará la segregación de edad y número de cotizaciones, pero cuyo resultado aplica en general.

En los cuadros 25 y 27 del informe IVM-UCR (Arias et al., 2016, p. 64, p. 66), se presentan los datos de la CCSS con los 1.570.422 afiliados cotizantes (en el último mes de cotización), y los 1.175.472 personas afiliados no cotizantes, para el 2015. Si se cuenta el total de afiliados cotizantes y se divide entre el total de afiliados $(2.745 .894=1.570 .422+1.175 .472)$, se obtiene la probabilidad de que un afiliado (sin importar la edad o las cotizaciones) sea cotizante para el 2015, es decir, $P=57,2 \%$.

Para minimizar la magnitud del error en los cálculos del equipo de la Escuela de Matemática (Arias et al., 2016), si se asume que en el cuadro No. 25 de Arias et al. (2016) aparece una persona en el mes " $\mathrm{i}>0$ ", entonces ésta cotizó continuamente desde el mes 1 hasta el mes "i" calcular el número esperado de cotizaciones de los afiliados, se procede a multiplicar el número del mes (columna 1, cuadro No. 25) por la cantidad de personas que cotizaron en ese mes por última vez (columna 8, cuadro No.25), y se divide por el total de afiliados, es decir, se esperan 6,39 cotizaciones al año entre afiliados. Si se repite este ejercicio, pero se le divide el total de cotizantes en lugar de afiliados, se obtiene que se esperan 11,17 cotizaciones al año entre los cotizantes. Si se ve esto en proporción anual (dividiéndolos por 12), se obtiene que $D^{U C R}=53,2 \%$, y $D^{\text {Modelo }}=93 \%$. Verificando la relación (3) se tiene,

$$
D^{U C R}=53,2 \%=57,2 \% \cdot 93 \%=P \cdot D^{\text {Modelo }}
$$

Si se usara $D^{U C R}$ como probabilidad de que un afiliado cotice, se tendría que en el 2015 el número de cotizantes sería,

$$
A f \cdot D^{U C R}=2.745 .894 \cdot 53,2 \%=\underbrace{1.461 .293}_{\text {Incorrecto }}<\underbrace{1.570 .422}_{\text {Correcto }}=2.745 .894 \cdot 57,2 \%=A f \cdot P
$$

Claramente, el número de "cotizantes" obtenido con la densidad de cotización de afiliados es inferior a la verdadera cantidad obtenida con la probabilidad de ser cotizante. Aún más, la diferencia entre ambas poblaciones es de 109.129 personas (un 7\% de los cotizantes reales). Si se considera que en el 2016 ingresaron 186.079 personas (nuevas generaciones), se torna obvio que la cantidad de nuevos afiliados se reduciría a la mitad, con las probabilidades correctas.

Esto afecta directamente a las fórmulas (5) y (6). En vista de la ecuación (1), la cantidad correcta de cotizantes sobrevivientes con edad " $\mathrm{x}+1$ " $y$ " $t+1$ " años de cotizar, debería ser

6 Si las cotizaciones no son continuas, entonces las probabilidades a calcular se alejan aún más entre sí. 


$$
\mathrm{E}\left[\operatorname{Cot} S_{x+1, t+1, n+1}\right]=\overbrace{\underbrace{A f_{x, t, n} \cdot P_{x, t, n}}_{C o t_{x, t, n}} \cdot D_{x, t, n}^{\text {Modelo }}}^{A f_{x, t, n} \cdot D_{x, t, n}^{U C R}} p_{x}^{\tau}+\overbrace{\underbrace{A f_{x, t+1, n} \cdot P_{x, t+1, n}}_{C o t_{x, t+1, n}} \cdot\left(1-D_{x, t+1, n}^{\text {Modelo }}\right) p_{x}^{\tau}}^{\text {Olvidado por la UCR }}
$$

En palabras esto se leería así: la cantidad esperada de cotizantes de edad " $\mathrm{x}+1$ " y con " $\mathrm{t}+1$ " años de cotizar, sería la proporción de afiliados que el año anterior tionen edad " $x$ " $y$ " $t$ " años de cotizar, que son cotizantes durante ese año (por eso se le multiplica $\left.P_{x+n}\right), y$ cuyas cotizaciones son suficientes para acceder al estado de " $t+1$ " años de cotizar; más aquellos con " $t+1$ " años de cotizar, que también son cotizantes ese año, pero que no alcanzan el número de cotizaciones para pasar al grupo con " $t+2$ " años de cotizar, tal y como lo indica el diagrama de Lexis asociado a este modelo.

El balance final sería que hay una cantidad importante de cotizantes que no serían considerados dentro del modelo del equipo de la Escuela de Matemática. Así, la cantidad de cotizantes sobrevivientes reales sería menor que el estimado por el la Escuela de Matemática en el informe IVM-UCR (Arias et al., 2016). A primera vista pareciera que esto vendría a inflar los ingresos, pero se hace la observación de que los ingresos están determinados por la cantidad de cotizantes (porcentaje de la PEA determinado a priori), y no por los cotizantes sobrevivientes. El dato de cotizantes sobrevivientes se utiliza a la hora de calcular las nuevas generaciones (Arias et al., 2016, p. 243), a través de la siguiente fórmula,

$$
N G_{n}=\frac{\overbrace{\sum_{x \geq 0} \operatorname{Cot}_{x, n}-\sum_{x, t \geq 0} \mathrm{E}\left[\operatorname{Cot} S_{x, t, n}\right]}^{\text {Sobreestimado }}}{p_{0,5}^{\tau}}
$$

Se enfatiza en el hecho de que la Escuela de Matemática contaba con la información necesaria para calcular las probabilidades de cotizar, y corregir así este sesgo. Por ejemplo, con la información presentada en el informe IVM-UCR (Arias et al., 2016) es posible hacer algunas estimaciones globales de la probabilidad de ser cotizante.

Utilizando el Cuadro No. 23 del informe IVM-UCR (Arias et al., 2016, p. 63) con el registro de los afiliados cotizantes del 2015, y el Cuadro No. 27 del informe IVM-UCR (Arias et al., 2016, p. 66) de los afiliados no cotizantes del 2015, segregado por género y edad, se logra obtener una probabilidad de ser cotizante, pero sin diferenciar por años de cotización. Con estas probabilidades "proxy" se podrían calcular de mejor manera los cotizantes sobrevivientes $\left(\mathrm{E}\left[\operatorname{Cot}_{x, t, n}\right]\right)$, y con ellos se estimarían las nuevas generaciones de una forma más precisa.

\section{Población implícita generada}

Como se especificó anteriormente, la imprecisión de utilizar la "densidad de cotización" como si fuera la "probabilidad de ser cotizante" reduce la base de "cotizantes sobrevivientes" e incrementa la base de "nuevas generaciones". Además, dado que se agrega la PEA y las nuevas generaciones, distribuyéndolas de manera constante para todos los años, surge un problema de consistencia demográfica en el modelo. Para apreciar el impacto de estos sesgos en el modelo del informe IVM-UCR (Arias et al. (2016), se requiere hacer uso de relaciones demográficas que indiquen las poblaciones que asume (implícitamente) el modelo, a partir de los datos generados.

Sea $\mathrm{PEA}_{\mathrm{n}}(15,19)$ la población económicamente activa con rango de edades entre 15 y 19 años, para el n-ésimo año, $y \operatorname{PEA}_{n}(k)$ la población económicamente activa con edad " $k$ " para el n-ésimo año. Dado que para este grupo es imposible la jubilación, entre un año y otro, existiría una salida de 
$\operatorname{PEA}_{\mathrm{n}}(15,19)$ por causa de muerte (con probabilidad qm) o invalidez (con probabilidad $\mathrm{q}_{\mathrm{i}}$ ), o porque tenía 19 años y sobrevivió un año más (saliendo de ese grupo de edad). Esto establece que si $\mathrm{NG}_{\mathrm{n}}(\mathrm{k})$ es la cantidad de personas que ingresan a la PEA con edad "k" para el n-ésimo año, entonces,

$$
P E A_{n}(15,19)=P E A_{n}-1(15,19) \cdot p-\text { Salientes }_{n}(19 \rightarrow 20)+N G_{n}(15,19)
$$

donde $N G_{n}(15,19)=\left(N G_{n}(15)+N G_{n}(16)+N G_{n}(17)+N G_{n}(18)+N G_{n}(19)\right)$ son los nuevos ingresos del grupo de edad entre 15 y 19 años, $p^{\tau}=\left(1-q_{m}\right)\left(1-q_{i}\right)$ es la probabilidad de sobrevivir a ambos decrementos, y Salientesn $(19 \rightarrow 20)$ son los sobrevivientes que salieron del grupo por cumplir 20 años.

Si se conocen los valores de $P E A_{n}(15,19)$ y de los nuevos ingresos a $N G_{n}(15,19)$, entonces se puede deducir el número de personas que salen cada año de este grupo de edad por cumplir 20 años, simplemente despejando el valor Salientes $_{n}(19 \rightarrow 20)$ de la ecuación (9). Aún más, considerando las probabilidades de sobrevivencia, se puede conocer la población económicamente activa con 19 años para el año anterior a partir de la relación:

$$
\text { Salientes }_{n+1}(19 \rightarrow 20)=\operatorname{PEA}_{n}(19) \cdot p^{\tau}
$$

De esta forma, conociendo la $P E A_{n}(k+1)$ y $N G_{n}(k+1)$, se puede deducir la población económicamente activa con " $k$ " años, a partir de la relación:

$$
P E A_{n+1}(k+1)=\underbrace{P E A_{n}(k) \cdot p^{\tau}}_{\text {sobrevivientes }}+\underbrace{N G_{n+1}(k+1)}_{\text {entrantes }}
$$

En general, utilizando estas relaciones recursivamente, es posible deducir las poblaciones económicamente activas, para cada edad, a partir de la fórmula:

$$
P E A_{n}(k)=\frac{\text { Salientes }_{n+20-k}(19 \rightarrow 20)}{\left(p^{\tau}\right)^{20-k}}-\sum_{l=1}^{19-k} \frac{N G_{n+20-k-l}(20-l)}{\left(p^{\tau}\right)^{20-k-l}} ; k=15,16,17,18,19 .
$$

\section{Población comparativa inflada}

Una vez identificadas varias de las deficiencias técnicas del modelo, es posible proceder a cotejar los valores generados por el mismo a niveles agregados, en aras de cuantificar las consecuencias en las proyecciones de los afiliados (base sobre la cual se generan los futuros pensionados). En efecto, la cantidad de afiliados que el modelo genera sobrepasa cualquier realidad posible. La justificación que se ha dado al respecto se sustenta en dos frentes que se presentan en el informe IVM-UCR (Arias et al., 2016, p. 167):

- Afiliados y pensionados extranjeros: de acuerdo al informe del equipo de la Escuela de Matemática, la totalidad de los afiliados al IVM no pueden ser comparados con la población 
nacional ni con la PEA, esto por cuanto las dos últimas excluyen a las personas que no están residiendo en el país, mientras que el IVM incluye a cualquier afiliado o pensionado de este régimen sin importar su lugar de residencia o nacionalidad. Esto es parcialmente correcto $^{7}$; sin embargo, es posible modificar las poblaciones nacionales o la PEA para que se vuelvan "comparables", sirviendo de cota superior para la población de afiliados.

- Pensionados con más de una pensión: en el mismo informe se explica que el IVM permite que existan personas pensionadas con más de un riesgo, y dan como ejemplo a una persona que sea pensionado directo, al mismo tiempo que recibe una pensión por viudez. Esto provoca que, por cada individuo con doble pensión, por ejemplo, se deba contar una persona más en los pensionados que no existe en la población nacional. Esto también es correcto, y se puede estimar la cantidad de personas en esta situación según el modelo. Se debe mencionar que es realmente raro encontrar a personas con más de una pensión (en el 2015 representaban el 2,7\% de todos los pensionados), y es aún menos probable que disfrute de tres pensiones.

Estos argumentos se toman en consideración al efectuar las comparaciones pertinentes. El informe IVM-UCR de la Escuela de Matemática define "afiliado" como "aquellos que tienen al menos una cuota ingresada, que están vivos y no pensionados" (Arias et al., 2016, p. 62). De este modo, es posible afirmar que la población de afiliados no se ve afectada por la segunda explicación, ya que los afiliados no incluyen al grupo de los pensionados. Por ejemplo, si una persona goza de una, dos o tres pensiones, pero a la vez está trabajando y cotizando para el IVM (en su pensión de vejez), entonces dentro de los afiliados solo se contaría una única vez.

De esta forma, el único problema que se enfrenta con los afiliados radica en aquellos que viven en el extranjero. Para presentar un escenario favorable para los cálculos sesgados del equipo de la Escuela de Matemática presentados en Arias et al. (2016), se construye una población INECCCP "inflada", que debe ser más grande (forzosamente) que la totalidad de los afiliados al IVM, y, de hecho, tendrá más personas que el IVM en general (incluyendo pensionados).

- Supuesto 1 - Inmigrantes: al 2015, todos los extranjeros afiliados deciden irse del país (dejando de ser parte de la población nacional y de la PEA). Aún más, del 2016 en adelante, todos los extranjeros que ingresan al país cotizan al menos una vez al IVM (100\% de cobertura para ellos) e inmediatamente se van de Costa Rica, por lo que no son contabilizados en la población nacional, pero sí dentro de los afiliados al IVM.

- Supuesto 2 - Emigrantes: todos los costarricenses que viven en el exterior, cotizaron al menos una vez al IVM (100\% de cobertura para ellos, aunque haya menores de 15 años), es decir, no están en la población nacional pero sí en el IVM.

- Supuesto 3 - Inmortalidad: todos los migrantes (extranjeros que cotizaron y se fueron, o costarricenses que viven en el exterior) nunca mueren, de modo que serán contabilizados en todos los años sin considerar la posibilidad de muerte.

7 Inversamente, hay personas en la PEA y en la Población Nacional, pero que nunca han cotizado al IVM.

8 El Centro Centroamericano de Población (CCP) estimó que podrían haber poco más de 300.000 costarricenses en el exterior, y el Instituto Nacional de Estadística y Censos (INEC) estimó (en el Censo del 2011) que esta cifra rondaba los 114.000 personas. Se toman 400.000 como cota superior, para estar seguros de incluir a todos los que viven en el extranjero.

$9 \quad$ El 15\% de los afiliados al IVM son extranjeros (Arias et al., 2016, p. 167).

10 Se toman estos datos de los estimados por el INEC y el CCP (INEC-CCCP, 2013) que están proyectados hasta el 2100 . En físico solo se imprimieron las proyecciones hasta el 2050, pero es posible acceder a esta información en el sitio web: https://censos.ccp.ucr. ac.cr/index.php/censos_c?censo=crproyna. 
Siguiendo estos supuestos, claramente inviables, a la población nacional del año "n", se le añaden 400.000 costarricenses en el exterior $^{8}$, se le suman los 411.884 de extranjeros en el IVM al $2015^{9}$, y se le agrega el acumulado de todas las migraciones desde el 2016 hasta el año "n"10.

Se hace énfasis en el hecho de que la población de afiliados dentro del IVM no puede superar esta población INEC-CCP "inflada", ya que esta contiene toda la población existente en el país, y le agrega los casos que cuentan en el IVM y no en la población.

\section{RESULTADOS}

Antes de iniciar con el análisis de los resultados, es importante hacer notar que en el informe IVM-UCR de la Escuela de Matemática de la UCR (Arias et al., 2016) se mencionan los riesgos asociados a un régimen de pensiones, entre los cuales se citan:

"Riesgo actuarial o de modelación: riesgo derivado del uso de modelos de valoración y supuestos (por ejemplo, hipótesis de mortalidad, longevidad, natalidad, discapacidad, inflación, tasa de interés y cobertura)." (Arias et al., 2016, p. 25).

Aún más, en dicho documento se citan los lineamientos de la Asociación Internacional de Seguridad Social (AISS) sobre la "buena gobernanza", y en la página 33 se cita el ISAP1 y el ISAP2 de la Asociación Internacional de Actuarios (IAA, por sus siglas en inglés), sobre los estándares para las prácticas actuariales. Entre otros, también hacen referencia a los estándares para las prácticas actuariales de la Actuarial Standards Board y el Financial Reporting Council (2010).

Respecto a la importancia de validar el modelo a utilizar, las hipótesis, $y$ los datos que lo alimentan, en aras de minimizar el riesgo de modelación, las entidades anteriormente mencionadas expresan lo siguiente:

Asociación Internacional de Actuarios: respecto a los datos preparados por terceros (como la población económicamente activa, PEA, entregada por la CCSS al equipo de la Escuela de Matemática), el ISAP1 propone:

"Validation - The actuary should take reasonable steps to review the consistency, completeness, and accuracy of the data used. These might include: testing the data for internal consistency." (International Actuarial Association [IAA], 2012, p. 6).

Aún más, en noviembre del 2016 se emitió el ISAP1A, actualización que incluye el riesgo de modelación. En este se aprecia lo siguiente:

"The actuary involved in selecting, modifying, developing, or using models should:

1. Be satisfied that there is in place an appropriate model risk management framework that addresses identification of model risks, assessment of these risks, and appropriate actions to mitigate these risks such as adequate model validation, documentation, and process controls.

2. Be satisfied that an appropriate model validation has taken place. Items that the actuary should consider, if applicable, include the availability, granularity, and quality of data and inputs required by the model, the appropriateness of the relationships recognized, and the model's ability to generate an appropriate range of results around expected values." (International Actuarial Association [IAA], 2016, p. 2)

Actuarial Standards Board: en setiembre del 2013, fue presentada una guía llamada Exposure Draft (2013) donde se lee: 
The actuary should attempt to mitigate model risk using validation, checking, analysis, governance and controls... Validation of the model could include

1. a reconciliation of relevant output values to actual data...

2. testing the model projection results against historical data to verify that modeled results bear a reasonable relationship to actual results over a given time period. (Actuarial Standards Board, 2013, p. 7).

Financial Reporting Council: en abril del 2010 emitió la referencia llamada Technical Actuarial Standard Modelling, donde incluyen principios sobre la "validación de los modelos", así como los datos y las hipótesis utilizadas. Entre ellos se encuentran los siguientes:

"The explanation of how the model is a satisfactory representation can be supported by techniques such as:

1. comparing the outputs of realizations with actual experience.

2. quantitative analysis of the predictive properties of the model using back-testing ${ }^{11}$ " (Financial Reporting Council, 2010, p. 9).

De acuerdo a estos principios, entre los deberes de un actuario se encuentran: validar los datos a utilizar, testear el modelo, sus ajustes, y sus predicciones, así como las hipótesis empleadas. Las deficiencias encontradas se pueden clasificar dentro del "Riesgo de Modelación", concretamente en tres áreas específicas: "Riesgo de Datos Utilizados", "Riesgo de Modelación Matemática (implementación y ajuste)", y "Riesgo de Hipótesis".

Para el desarrollo de esta sección se hace uso de las herramientas desarrolladas en la sección de metodología y se cuantifica el impacto de los tres sesgos de modelación observados en esta revisión. La primera subsección lidia con el primer tipo de sesgos (riesgo de datos utilizados), caracterizándolo y explicando las razones del por qué se considera que se usaron datos incorrectos. La segunda subsección se enfoca en el modelo implementado, así como su ajuste (segundo tipo de sesgo). En la última subsección, se analizan las deficiencias encontradas en algunas de las hipótesis (tercer tipo de riesgo), tales como el salario de referencia o la composición sectorial. En la primera subsección se utiliza el modelo de la UCR (Arias et al., 2016) para analizar ambas poblaciones económicamente activas, y para la segunda subsección se utiliza una sola fuente de datos, pero bajo el modelo de la UCR y el modelo mejorado, de modo que se analiza el impacto de este sesgo.

\section{Riesgo de datos utilizados: PEA}

La base demográfica del estudio IVM-UCR (Arias et al., 2016) presenta inconsistencias. Dicha base es la población económicamente activa proyectada al futuro. En dicho estudio se especifica que "se utiliza la PEA proporcionada por la CCSS" (Arias et al., 2016, p. 59), la cual se presenta agregada en la sección 9.8 de Anexos de dicho documento. Esta PEA-CCSS no coincide con las proyecciones más confiables, tales como las que se derivan de utilizar la población oficial (INEC-CCP) junto con hipótesis razonables sobre las tasas de actividad (PEA-INEC-CCP), o las generadas por la CELADE (2017).

11 Este test podría haber sido implementado de la siguiente forma: se utilizan datos desde 1975 hasta el 2000 para ajustar el modelo, se proyecta la población de cotizantes y pensionados hasta el 2015, y se comparan contra los datos observados (validando el modelo). Precisamente esto fue lo que se solicitó al equipo de la Escuela de Matemáticas, pero lamentablemente no fue realizado. 
Cabe enfatizar que el efecto directo de utilizar esta PEA-CCSS es claro, principalmente porque la misma subestima la cantidad de trabajadores y desempleados que existirán en el futuro próximo, pero a partir del año 2073, las sobreestima. De hecho, se estarían subestimando los ingresos que recibiría el IVM durante los primeros 60 años del estudio, afectando claramente las finanzas y la sostenibilidad del fondo en cuestión. Dado que los puntos de quiebre, según los resultados del estudio IVM-UCR (Arias et al., 2016), se encuentran antes del 2030, resulta importante lo que suceda en el corto plazo, siendo relativamente irrelevante la sobreestimación que se presenta en el largo plazo.

Se inicia realizando test cruzados para probar la validez de esta PEA-CCSS, enfatizando en las contradicciones demográficas que ella encierra. Se compara contra la PEA-INEC-CCP, y se contrasta la población generada por el modelo con ambas PEAs, confirmando la subestimación de ingresos del IVM por parte del informe IVM-UCR (Arias et al., 2016).

\section{"PEA-CCSS"Vs "PEA-INEC-CCP”}

A continuación, se contrastan los valores de la PEA-CCSS contra los de la PEA-INEC-CCP. Como se muestra en el gráfico 1, la proyección de la CCSS resulta en cifras de PEA menores que las que probablemente tendrá Costa Rica en el corto y mediano plazo.

\section{GRÁFICO 1 \\ COMPARACIÓN DE POBLACIONES ECONÓMICAMENTE ACTIVAS}

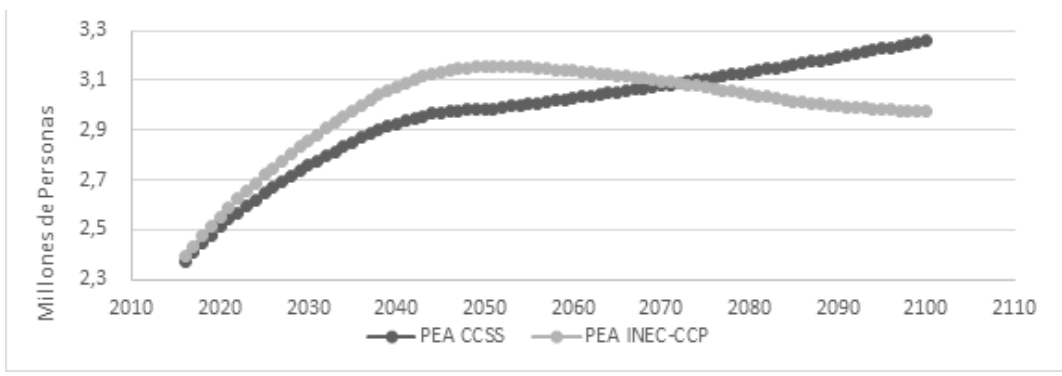

Fuente: PEA CCSS proviene de datos obtenidos de la PEA generada por la CCSS, publicados por esa entidad en el 2014. PEA INEC-CCP es elaboración propia basada en los datos publicados del INEC y el CCP.

Por otra parte, después del 2050, la PEA-CCSS, al crecer geométricamente, superará a la PEA-INEC-CCP de la proyección más fiable hacia el año 2072. Para el año 2050 la CCSS proyectó una PEA que es menor en 167.000 personas que la proyección más probable (PEA-INEC-CCP). En una fecha más cercana, el año 2030, la CCSS espera una fuerza de trabajo con 99.000 personas menos que las que probablemente habrá ese año. Esto significa que se está subestimando las cantidades de cotizantes al sistema IVM y, por tanto, exagerando las dificultades del régimen en el corto y mediano plazo. En contraste, para el largo plazo (después de 2050) se está proyectando un aumento de trabajadores que probablemente no tendrá lugar.

Es interesante notar como la PEA-INEC-CCP empieza a decrecer después del 2050, en concordancia con el comportamiento esperado de la población mayor a 15 años, mientras que la PEACCSS presenta un crecimiento sostenido después de esa fecha. Si bien es cierto que esta diferencia en tendencias es incómoda, no es suficiente para aseverar que existe un error en las proyecciones, por lo que se procede a comparar los datos de esta PEA-CCSS contra la población en edad laboral para analizar su consistencia.

\section{Población en Edad de Laboral Vs PEA-CCSS}


En el gráfico 2 se compara la población entre 15 y 64 años de la "población oficial" (INECCCP) y de la "población alternativa" (CELADE, 2016) contra la PEA-CCSS en ese mismo rango de edades. Se hace el recordatorio de que en este artículo académico se está considerando la población del INEC-CCP como la oficial debido a que cuentan con estadísticas más precisas a la hora de su confección, en comparación con la generada por la CELADE en su informe del 2016.

GRÁFICO 2

\section{POBLACIONES PROYECTADAS ENTRE 15 Y 64 AÑOS Y PEA-CCSS}

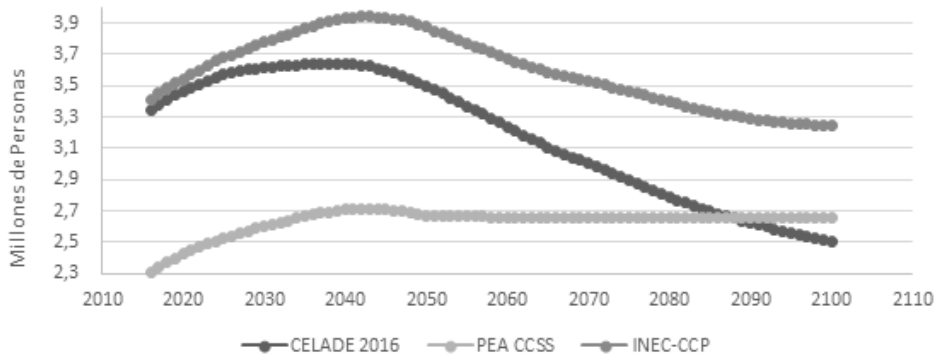

Fuente: PEA CCSS proviene de datos obtenidos de la PEA generada por la CCSS, publicados por esa entidad en el 2014. Los datos poblacionales CELADE 2016 y INEC-CCP fueron tomados de la versión 2016 del informe de la CELADE, y de los datos publicados del INEC y el CCP, respectivamente.

Como se mencionó antes, si se utilizara la población de la CELADE (2016), automáticamente se estaría invalidando la PEA-CCSS por contradecir una regla demográfica inviolable, al ser más grande que la población total en ese rango de edades. Para poner esto en perspectiva, de acuerdo a la PEA-CCSS, para el año 2087, todo ciudadano que habita en el país, con edades entre 15 y 64 años, sería un trabajador o está buscando trabajo, ninguno podría estudiar ni estaría pensionado. Aún más, ya para el 2100, la PEA-CCSS nos indica que existen más personas buscando trabajo o trabajando, que la cantidad posible de personas en esta situación, lo cual es contradictorio. Dicho esto, se procede a contrastar la PEA-CCSS contra la población oficial del INEC-CCP (2013).

Aún para la población oficial se presentan contradicciones demográficas para algunos grupos poblacionales. Por ejemplo, si se toma la población total de hombres con edades entre 60 y 64 años de edad, $y$ se compara contra la PEA-CCSS para ese grupo de edades, se nota que la segunda supera a la primera para el año 2100 (ver gráfico 3). Para ese año la PEA-CCSS posee 28.000 personas más que la población total dentro de ese rango de edades para la población alternativa de CELADE (2017), y contiene 673 personas de más que la población total en ese intervalo según la población oficial del INEC-CCP.

\section{GRÁFICO 3 \\ POBLACIONES PROYECTADAS Y PEA-CCSS ENTRE 60 Y 64 AÑOS}

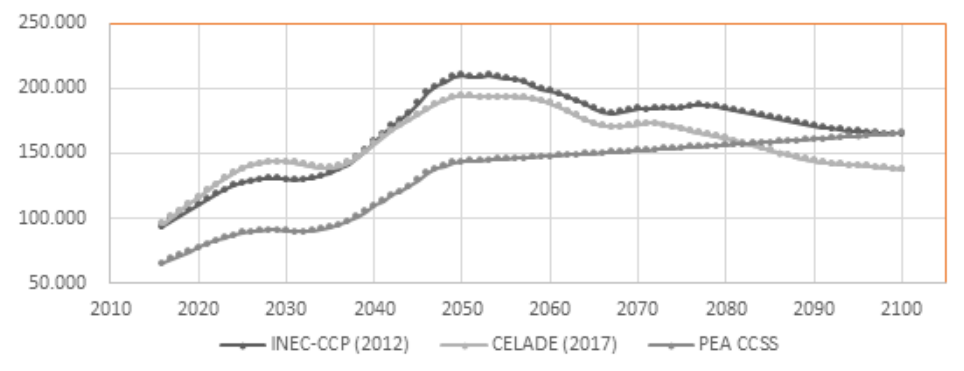

Fuente: PEA CCSS proviene de datos obtenidos de la PEA generada por la CCSS, publicados por esa entidad en el 2014. PEA INEC-CCP es elaboración propia basada en los datos publicados del INEC y el CCP. 
Aún más, al estudiar las tasas de actividad implícitas dentro de los valores presentados en la PEA-CCSS, se observa que se da un cambio inexplicable a partir del año 2050. En el gráfico 4 se presentan las tasas de actividad implícitas, derivadas de la PEA-CCSS y la población INEC-CCP para las edades entre 60-64 años y se hace evidente la manera como pasa de mantener un nivel estable de 70\% hasta el 2050, para empezar a crecer estrepitosamente hasta alcanzar poco más del 100\% en el 2100, algo imposible en la realidad. Es notorio que las personas en este grupo de edades están próximas a la jubilación, o ya están jubiladas, razón por la cual se tornan inverosímiles estas proyecciones.

\section{GRÁFICO 4 \\ TASAS DE ACTIVIDAD (IMPLÍ́CITAS) DE HOMBRES ENTRE 60 Y 64 AÑOS}

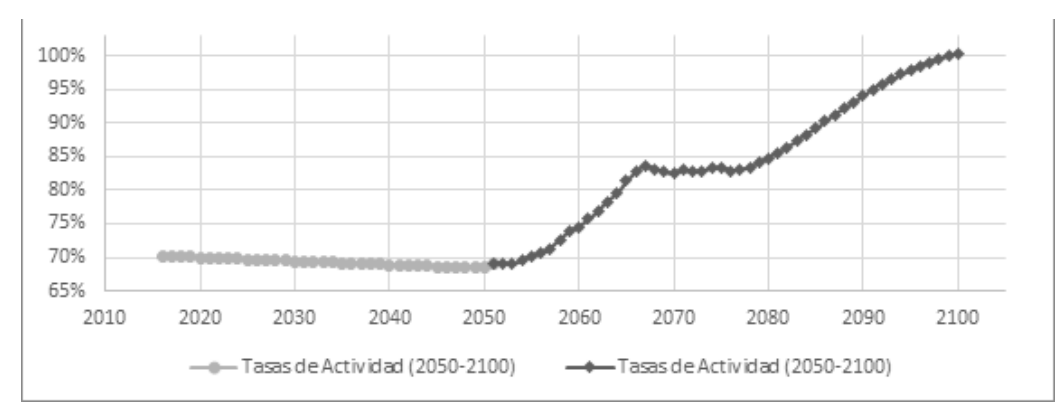

Fuente: Elaboración propia basada en la PEA de la CCSS y la población del INEC-CCP.

\section{Cotizantes proyectados}

Para calcular el impacto de la subestimación de la PEA-CCSS (en el corto plazo) sobre los afiliados que cotizan, se comparan los cotizantes que arroja el modelo de la UCR usando la PEA-CCSS (cotizantes UCR) contra los cotizantes generados bajo la PEA-INEC-CCP (cotizantes INEC-CCP). En el gráfico 5 se hace esta comparación, y se aprecia que inicialmente ambos son muy similares, pero conforme pasa el tiempo, la diferencia se empieza a hacer notar, hasta que para el año 2050 la discrepancia ya es de 116.698 cotizantes, un nivel nada despreciable.

\section{GRÁFICO 5 \\ COTIZANTES PROYECTADOS}

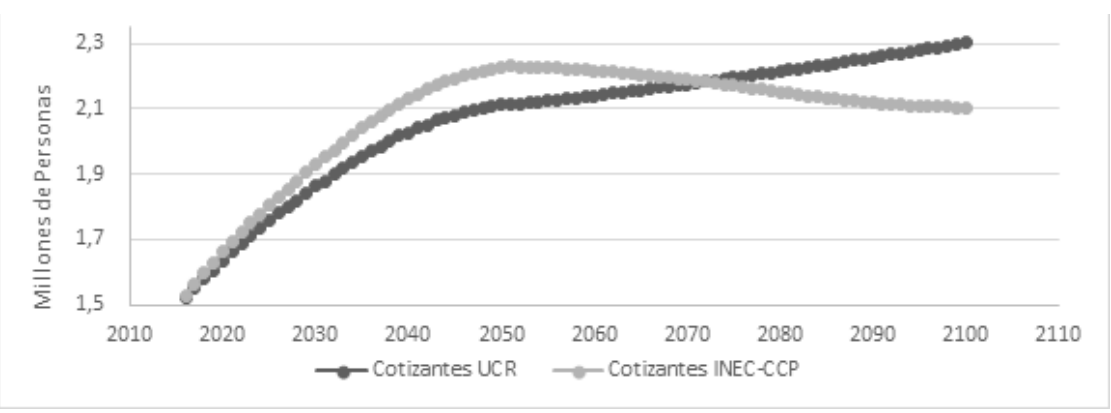

Fuente: Cotizantes UCR son tomados del informe IVM-UCR (Arias et al., 2016). Cotizantes INEC-CCP es elaboración propia basada en el modelo de la UCR, pero alimentado con la PEA del INEC-CCP. 
Para dimensionar esto, se calcula lo que representaría este déficit de cotizantes dentro de las finanzas del IVM. Según los datos utilizados por Arias et al. $(2016)^{12}$, para el 2015 se cuenta con un salario promedio (ponderado por sector y sexo) de alrededor de 482.835 colones. A continuación, se utiliza este salario promedio para realizar los cálculos, aunque se sabe que se está subestimando el impacto del sesgo, debido a que no se está considerando el aumento real de los salarios a lo largo del tiempo (causado por el aumento en la productividad). De modo que, si se multiplica este salario promedio por la cantidad de cotizantes que no se están considerando en el 2050, usando 10,5\% de prima para ese año, se concluye que se estaría dejando de recibir un total de 70.571 millones de colones (del 2015) en todo ese año, lo cual es una cantidad suficientemente elevada como para afirmar que la subestimación en la población de cotizantes es un sesgo relevante.

\section{Cobertura: "Cotizantes UCR" Vs "PEA-INEC-CCP"}

Habiéndose efectuado este análisis previo, se está en posición de comprender los hallazgos obtenidos al calcular los porcentajes de cobertura de los cotizantes generados en el informe IVM-UCR (Arias et al., 2016), respecto de la PEA-INEC-CCP. Las hipótesis utilizadas por Arias et al. (2016) para los distintos escenarios se pueden encontrar en la página 3 del informe IVM-UCR (Arias et al., 2016).

Primero, nótese que los cotizantes generados por el modelo con la PEA-CCSS son estrictamente crecientes (salvo en un año), mientras que la PEA-INEC-CCP empieza a decrecer en el 2050, dando como resultado que los cotizantes generados con la PEA-CCSS empiezan a alcanzar a la PEAINEC-CCP en el largo plazo. Sin embargo, en el corto plazo el efecto que domina es la subestimación de la población.

Segundo, verificando los porcentajes de representación, se nota que no se cumplió con la hipótesis de cobertura que se pretendía, puesto que un 70\% de la PEA-CCSS representa un porcentaje menor para la PEA-INEC-CCP. De hecho, si se comparan los porcentajes de "Cobertura Teórica" del cuadro No. 115 del informe IVM-UCR (Arias et al., 2016, p. 161) contra los porcentajes que representan los cotizantes sobre la PEA-INEC-CCP (Cobertura Real), se puede apreciar que la segunda fue mucho más baja que la primera en el corto y mediano plazo, y se torna mucho mayor en el largo plazo. De acuerdo a las hipótesis antes mencionadas, se debe alcanzar un nivel de cobertura del $70 \%$ en el año 2050, pero éste fue alcanzado por la Cobertura Real hasta en el año 2072, siendo que para el año 2050 el nivel de Cobertura Real era del 66\%. Para el 2100 el porcentaje de cobertura rondaba el 77\%, bastante mayor que el tope definido dentro de los supuestos. En el gráfico 6 se muestran las coberturas.

GRÁFICO 6

COBERTURA TEÓRICA VS. COBERTURA REAL

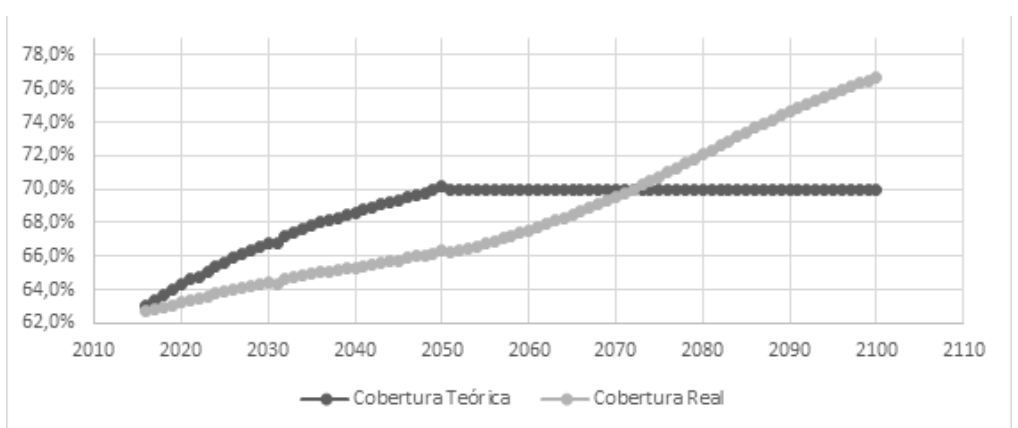

Fuente: La cobertura teórica fue tomada del informe IVM-UCR (Arias et al., 2016). La cobertura real es elaboración propia con los cotizantes del modelo de la UCR como porcentaje de la PEA del INEC-CCP.

12 Estos datos están en el Excel generado por Arias et al. (2016), facilitado a nosotros por la SUPEN. 


\section{Riesgo de modelación matemática: datos generados}

Es importante enfatizar que las observaciones sobre la densidad de cotización, nuevas generaciones y proyecciones de afiliados, tienen un impacto determinante sobre los resultados del estudio. Las tres presentan un exceso artificial en la base de afiliados, lo que impacta directamente a los pensionados futuros (quienes son una proporción de los afiliados), lo cual provoca que los costos se vean inflados artificialmente, inclusive en el corto plazo. Se podría sospechar que al aumentar los afiliados se aumentan también los cotizantes, por lo que existe un impacto análogo sobre los ingresos, pero este no es el caso ya que la forma en que se escogen a los cotizantes es a partir del porcentaje de cobertura de la PEA, es decir, los primeros se definen a partir de los valores estimados de los segundos (es una variable exógena dentro del modelo).

\section{Nuevas generaciones: PEA implícita}

Debido a que la pirámide poblacional se está invirtiendo, como se muestra en el gráfico No. 10 del informe IVM-UCR (Arias et al., 2016, p. 40), es incorrecto asumir que la distribución de las nuevas generaciones será constante durante los próximos 100 años. En vista de esto, se analiza la relación que existe entre las "nuevas generaciones" de afiliados con edades entre 15 y 19 años, y la PEA-CCSS para ese rango de edades. Inicialmente pareciera no haber problema, ya que las nuevas generaciones nunca sobrepasan a la PEA (aunque para la PEA-INEC-CCP mantiene niveles del 45\% de esta). Sin embargo, si se utiliza la PEA implícita por el modelo, como se explicó en la sección de metodología a través de la ecuación (12), se concluye que los números del informe IVM-UCR (Arias et al., 2016) no son cotejables con la realidad.

La probabilidad de invalidez más alta (para ambos sexos entre 2016 y 2050) en este grupo de edad es $\mathrm{q}_{\mathrm{i}}=0,00679 \%, y$ la de muerte es $\mathrm{q}_{\mathrm{m}}=0,07565 \%$. Para favorecer los cálculos sesgados del equipo de la Escuela de Matemática (Arias et al., 2016), se asume que todos en este grupo de edad tienen estas probabilidades para cada decremento ${ }^{13}$. Se utilizan los datos de la PEA-CCSS para el grupo de edades entre 15 y 19 años, así como la serie de nuevas generaciones para cada edad k (= 15, 16, 17, 18, 19) que genera el modelo implementado por el equipo de la Escuela de Matemática (Arias et al., 2016) ${ }^{14}$.

Dados los altos niveles observados en $\mathrm{NG}_{\mathrm{n}}(15,19)$ (alrededor de 37.000 personas por año durante la primera década - representa $41 \%$ de la PEA $\left._{n}(15,19)\right)$, la población implícita obtenida con la fórmula (12), para el rango de edades entre 15 y 17 años, sería negativa para varios periodos, lo cual se establece es una inconsistencia demográfica. En el gráfico 7 se muestran las nuevas generaciones implícitas con esta implementación que realizó el equipo de la Escuela de Matemática en el informe IVM-UCR (Arias et al., 2016).

13 Con esta hipótesis, se reduce la cantidad de personas con 19 años, permitiendo que haya más personas en las otras edades. Por esta misma razón, se ignora la probabilidad de dejar de trabajar, buscando que p sea máxima.

14 Las nuevas generaciones de la PEA son más altas que las de los cotizantes, por lo que al utilizar las segundas estamos favoreciendo los cálculos del equipo de la Escuela de Matemática, al reducir las personas que entran, y aumentando las personas en cada grupo de edad. 


\section{GRÁFICO 7 \\ PEA IMPLÍCITA GENERADA POR EL MODELO DE LA UCR}

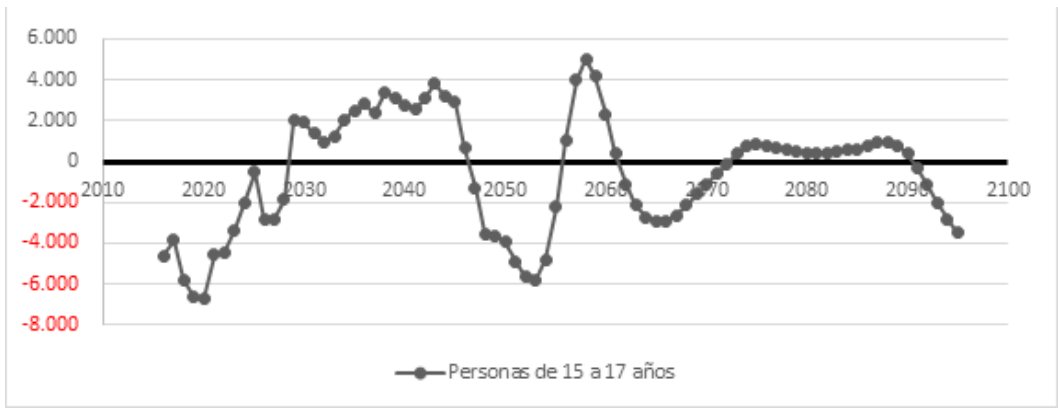

Fuente: Elaboración propia a partir de los cotizantes del modelo de la UCR y la PEA de la CCSS.

Claramente no es posible tener -4.628 personas con edades entre 15 y 17 años en el 2016. Este sesgo surge por utilizar datos consolidados de la PEA, a pesar de tener acceso a los valores segregados por grupos de edad quinquenales (15-19, 20-24, etc....). El algoritmo con datos agrupados es el que provoca que se pierda la relación entre las poblaciones totales y los subgrupos de edades que la conforman. Dado que utilizan una distribución entre edades constante, en años donde la $\mathrm{PEA}_{\mathrm{n}}(15,19)$ no sea suficientemente grande, las nuevas generaciones asignadas estarían siendo desproporcionales respecto de la población económicamente activa de ese grupo de edad. Esto se podría haber corregido si se utiliza el algoritmo anterior para cada grupo de edad quinquenal, utilizando una distribución (posiblemente constante) entre las 5 edades que lo conforman.

Los cálculos de la cantidad de los nuevos cotizantes del IVM se deben realizar de manera segregada por sexo y edad. En el estudio IVM-UCR (Arias et al., 2016) esos cálculos se hacen sobre el agregado total de la PEA, por lo que no se capturan los cambios en la estructura por edad de la fuerza de trabajo, que ciertamente van a ocurrir en Costa Rica. En la proyección PEA-INEC-CCP antes descrita, la edad media de la PEA pasa de 38,1 años en 2015 a 44,1 años de edad en 2050 y la proporción de trabajadores adultos mayores de 65 años aumenta de 2,6\% en 2015 a 9,1\% en 2050 . En la medida en que la prevalencia de cotizantes varía con la edad, el envejecimiento de la fuerza de trabajo tendrá consecuencias sobre las tasas de cotización y las distribuciones de las nuevas generaciones, efectos que no están siendo considerados en el estudio IVM-UCR (Arias et al., 2016).

\section{Proyección de afiliados}

Se emplea la población INEC-CCP "inflada", que se describe en la metodología del presente artículo, para efectos de comparar la población de afiliados que genera el modelo IVM-UCR (Arias et al., 2016). Se presentan los resultados en el gráfico 8. 


\section{GRÁFICO 8 \\ POBLACIÓN INFLADA VS. AFILIADOS}

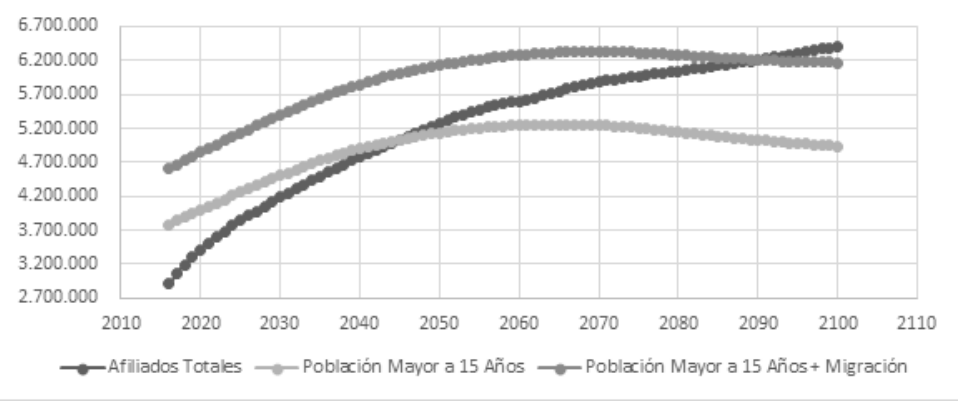

Fuente: Elaboración propia a partir de los afiliados del modelo de la UCR y población INEC-CCP Inflada.

De estos cálculos se desprende que, a pesar de todos los supuestos irreales que se tomaron para construir la población INEC-CCP inflada (inmortalidad de cotizantes, cobertura del 100\%, etc), el número total de afiliados (generado por el modelo) sobrepasa a la población mayor de 15 años más la migración acumulada, en el año 2089. A partir de esa fecha la diferencia solo crece y crece, hasta ser de 239.308 personas en el 2100. En términos matemáticos, esto es imposible, pues los afiliados al IVM no pueden superar a la población completa en edad laboral (incluyendo inmigrantes y nacionales viviendo en el exterior), especialmente cuando la cobertura del IVM dentro de la PEA no es del 100\% (existen muchos costarricenses que nunca en su vida han cotizado para el IVM).

Aún más, es improbable que todos los extranjeros afiliados al IVM, 411.884 personas, abandonen el país instantáneamente, o que todas aquellas personas que decidan emigrar a Costa Rica se queden un mes $y$ luego se vayan de nuestro país, sin contar que es claramente absurdo considerar que ninguno de ellos muere en 100 años. Esto significa que los datos poblacionales que se usaron en la proyección INEC-CCP inflada están muy sobrevalorados y, aun así, las proyecciones de afiliados del informe IVM-UCR (Arias et al., 2016) del equipo de la Escuela de Matemática supera esa cifra. De hecho, si se compara la base de afiliados contra la población nacional (una comparación más razonable que la anterior), se nota que los afiliados superan a la población en edad laboral a partir del año $2045^{15}$.

¿De dónde surgen los afiliados que no están incluidos en las migraciones ni en la población en edad laboral?

Si se agregan los pensionados (ver tabla 9.8.1 en Arias et al., 2016, p. 300), y se compara el IVM (afiliados más pensionados) contra la población inflada, la situación empeora, puesto que la cantidad de pensionados también son inflados, producto de la sobreestimación de los afiliados, por lo que el IVM llega a superar a la población total en el año 2045, y continúa su crecimiento hasta que en el 2100 ya representa el $134 \%$ de esta población.

Asumiendo que el IVM llega a cubrir a toda la PEA, a toda la población en edad laboral, y a todos los migrantes (nacionales y extranjeros), entonces el 34\% por encima de la población total, debería ser justificada por aquellos con dos, tres, o incluso cuatro pensiones, de acuerdo con la explicación presentada por el equipo de la Escuela de Matemática. Sin embargo, este 34\% representa un total de 2.094.480 personas, y según las proyecciones del equipo de la Escuela de Matemática, para este año sólo existen 1.855.172 pensionados, surgiendo un excedente de un cuarto de millón de personas sin justificación.

15 Los resultados empeoran si se comparan los afiliados contra la población, pero solamente considerando aquellos con edades entre 15 y 64 años. 
Estos resultados son incoherentes pues las 239.308 personas del IVM que no son ni pensionados, ni extranjeros afiliados, ni costarricenses afiliados viviendo en el exterior, ni personas en edad laboral, no pueden ser explicadas por el modelo, y es una inconsistencia a las relaciones demográficas que deberían respetarse. Nótese que se está comparando esto contra una población sobreestimada, lo que permite concluir que estas proyecciones están sesgadas, por lo que los resultados derivados de ellas son incorrectos.

\section{GRÁFICO 9 \\ POBLACIÓN INEC-CCP INFLADA VS. IVM}

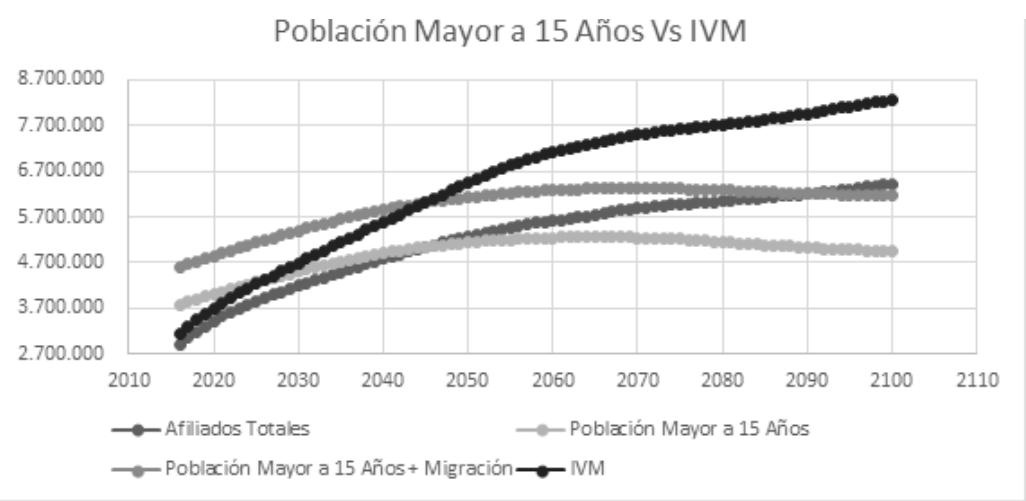

Fuente: Elaboración propia a partir del IVM del modelo de la UCR y población INEC-CCP Inflada.

Se puede realizar un ejercicio con datos más "realistas", utilizando la población oficial (INEC$\mathrm{CCP}$ ) en edad laboral sin "inflarla" artificialmente, y compararla contra el IVM. Se debe recordar que, aunque es cierto que hay personas que sí son contabilizadas en el IVM (como afiliadas o pensionadas) y no en la población nacional (viven en el exterior), de igual forma hay personas en la población nacional que no son contabilizadas en el IVM porque nunca en su vida han cotizado para ese régimen (ya sea porque no trabajan, o porque pertenecen a alguno de los regímenes sustitutivos, o simplemente no cotizan).

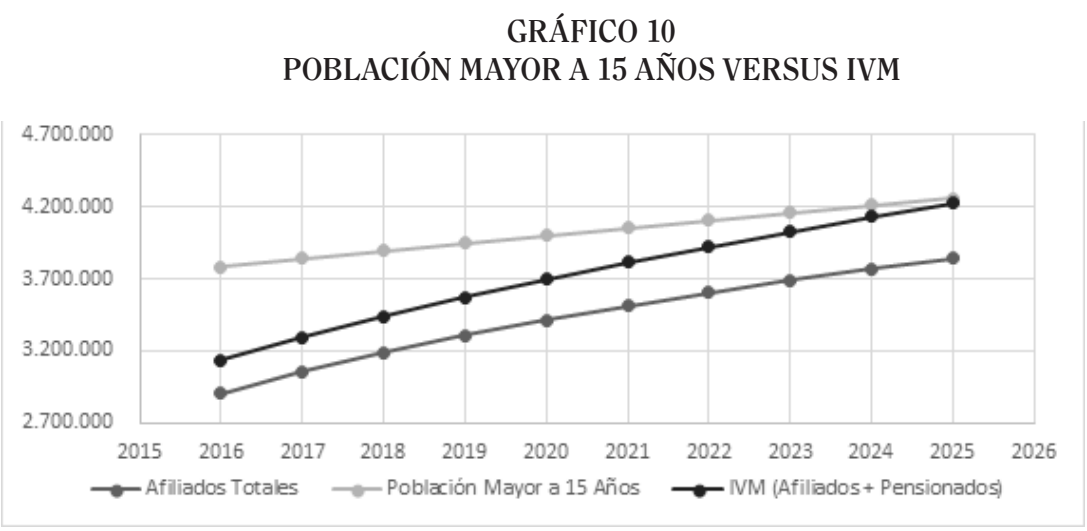

Fuente: Elaboración propia a partir del IVM del modelo de la UCR y población INEC-CCP. 
A partir de estos cálculos se obtiene que en una década los afiliados pasan de representar un $73 \%$ de la población en edad laboral en el 2015, a conformar el 90\% para el 2025. Peor aún, al agregar a los pensionados se observa que, en sólo una década, el IVM pasó de representar un 79\% de la población en edad laboral en el 2015, a alcanzar el 99\% durante el 2025, como se muestra en el gráfico 10.

Debe notarse que con esta cantidad de personas en el IVM se necesitaría una población de más de 7 millones de personas en el 2050 para que las cifras tengan un poco de sentido (para que el IVM no supere el $90 \%$ de la población total), pero esto excede en alrededor de 2 millones de personas el valor proyectado por el INEC-CCP. Por tanto, el impacto en el corto plazo es suficientemente fuerte como para influir en los resultados del estudio IVM-UCR (Arias et al., 2016), aumentando los costos del fondo de pensiones más no así los ingresos del mismo, sesgando los resultados en contra de su solvencia.

Finalmente, se podría argumentar que esto sucede por causa de la PEA-CCSS que se utilizó para generar los datos, la cual, como se mostró, estaba inflada en el largo plazo. Se calculan los afiliados que genera el modelo con la PEA-INEC-CCP, para ver si esto se corrige, sustentando la hipótesis de que el modelo está correcto y que el error proviene de los datos utilizados. Sin embargo, al realizar estos cálculos se obtiene que el modelo genera una base de afiliados que alcanza a la población total en el 2050, y luego se mantiene pegada al 100\% de la misma, como se muestra en el gráfico 11.

\section{GRÁFICO 11}

\section{POBLACIÓN MAYOR A 15 AÑOS VERSUS IVM CON PEA-INEC-CCP}

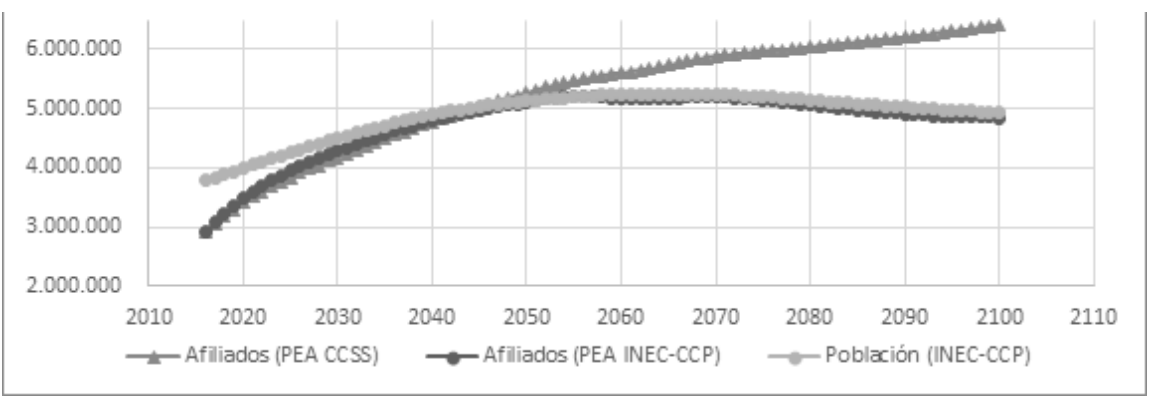

Fuente: Elaboración propia a partir del IVM del modelo de la UCR y población INEC-CCP.

Tomando en cuenta que según los datos de la PEA-INEC-CCP, las personas con edades altas llegarán a representar una parte importante de la población, tendría más sentido realizar esta comparación dentro de los rangos de mayor productividad de las personas, esto es, las edades entre 15 y 64 años. Al repetir los cálculos, pero filtrando por este grupo de edades, se hace claro que las proyecciones, aun utilizando una base de PEA fiable, resultan demográficamente contradictorias, como se muestra en el gráfico 12 . 


\section{GRÁFICO 12 \\ POBLACIÓN DE 15 A 65 AÑOS VERSUS IVM CON PEA-INEC-CCP}

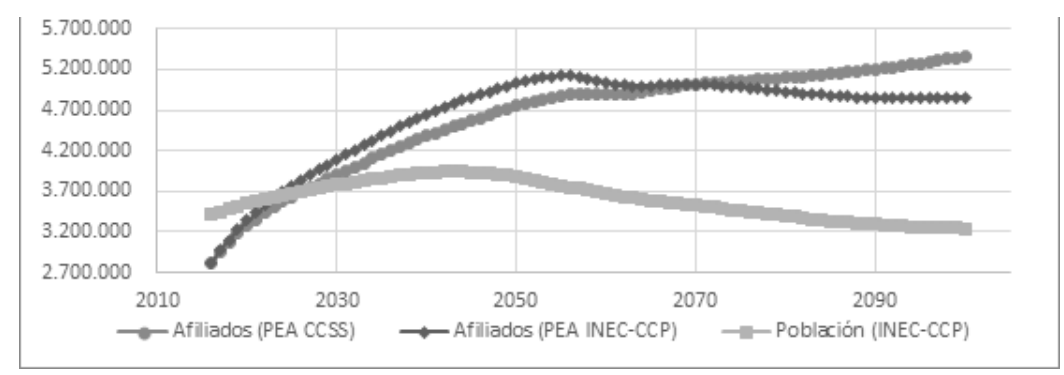

Fuente: Elaboración propia a partir del IVM del modelo de la UCR y población INEC-CCP.

Del gráfico 12 se observa que ya para el año 2023, los afiliados con edades entre 15 y 64 años alcanzaron a la población total esperada en ese mismo rango de edades. Peor aún, para el 2100 ya representa el 150\% de la población total, teniendo más de 1,6 millones de personas de exceso. Estas contradicciones provienen de la suma de todos los sesgos antes mencionados, tales como la sobreestimación de las nuevas generaciones (que infla a los afiliados), la distribución constante de estos nuevos afiliados, entre otros.

Se puede deducir de este análisis que la omisión del segundo término en la ecuación (7) provoca un incremento artificial en las nuevas generaciones que determina la cantidad de afiliados y pensionados que genera el modelo, aumentando así los costos del IVM, sin un respaldo técnico. Esto coincide con las conclusiones de la revisión técnica que realizó la Organización Internacional del Trabajo (OIT, 2017) al estudio actuarial IVM-UCR (Arias et al., 2016), donde se observaba que:

"1- El 33\% de los varones nacidos en el año 2000 estarían afiliados a la CCSS en el año 2020, el 68\% en el 2025 y el 90\% en el 2040; 2- Para la generación nacida en 1985, 118\% de los individuos estarían afiliados a la CCSS en el año 2030 y 121\% en el año 2040, es decir, la generación completa, no solo quienes trabajan; 3- La generación de los nacidos en el año 2020 estaría afiliada en un 114\% para el 2050; 4- El 53.7\% de toda la generación de mujeres nacidas en 1980, que alcanzan la edad de 60 años, llegan a obtener la pensión; 5- El 90.9\% de toda la generación de hombres nacidos en 1980, llegan a obtener la pensión; 6 - 85\% de la población de 65 años y más, logra obtener una pensión, pero solo alrededor de $2 / 3$ de la fuerza de trabajo, como máximo, está cubierta” (OIT, 2017, p. 3-4).

\section{Riesgo de hipótesis e implementación}

\section{Composición sectorial}

Respecto a la forma en que se definió la población a generar, el equipo de la Escuela de Matemática decidió agruparlos por género, sin considerar los sectores. En el informe IVM-UCR (Arias et al., 2016, p. 68) se afirma que no se tenía la información por sector para más de un año. Sin embargo, la información sí estaba disponible, debido a que contaban con la base de cuentas individuales (CI), donde se podía observar el sector al que pertenecía cada cotizante, como ellos mismos lo evidenciaron durante el análisis retrospectivo ${ }^{16}$. Más aún, cuando se analiza el comportamiento de los

16 Cuadro No 13 de IVM-UCR (Arias et al., 2016, p. 54) donde comparan cobertura según CI y Facturación. 
sectores, se concluye que son realmente distintos, tanto en crecimiento del salario real (Arias et al., 2016, p. 72), morosidad (Arias et al., 2016, p. 150), salario promedio (Arias et al., 2016, p. 71, 228, 229), entre otros. Ante esta realidad, resulta necesaria la división sectorial, en lugar de agruparlos.

El modelo del equipo de la Escuela de Matemática, se sustenta en tener salarios promedio por edad y sexo, ignorando las particularidades de los sectores productivos, entiéndase: público, privado, independiente, convenio, y doméstico. En el cuadro No. 115 del IVM-UCR (Arias et al., 2016, p. 161) se presentan los porcentajes de cobertura para cada escenario, mostrando un incremento desde $63,07 \%$ hasta el $70 \%$ en el escenario base. No parece, sin embargo, que se incluya dentro del análisis el sector del cual vendrán esos nuevos afiliados, ni el impacto dentro de la sostenibilidad del IVM por esta causa.

En la página 53 del informe IVM-UCR (Arias et al., 2016) se explica cómo se han modificado las representaciones de los sectores en la última década, y en el cuadro No. 12 del IVM-UCR (Arias et al., 2016, p. 53) se muestran los porcentajes de cobertura para cada sector, y su evolución desde el 2000 hasta el 2015. Ahí se logra apreciar que el sector público está "saturado", pues ya cotizan el 98,4\% entre todos los regímenes de pensiones de JUPEMA, IVM y Poder Judicial (Arias et al., 2016, p. 54). Además, los "cuenta propia" ya alcanzan el 88,8\% de cobertura, por lo que aumentar este sector sería difícil. Es en doméstico y privado donde existe una ventana para aumentar la cobertura, lo cual debería haber sido tomado en cuenta a la hora de considerar los efectos del incremento en esta variable. El informe IVM-UCR (Arias et al., 2016) inclusive lo expresa claramente cuando analiza el comportamiento de representatividad dentro del IVM:

"El aumento de los asegurados independientes y por convenios ha modificado la distribución por sector, como se observa en el Cuadro No 17. Mientras que éstos duplicaron su participación, los cotizantes del sector público la redujeron en 3 puntos porcentuales y los del sector privado en 9 puntos porcentuales" (Arias et al., 2016, p. 58).

Del gráfico 13 se desprende que la representatividad de los sectores dentro de los cotizantes del IVM ha cambiado, lo que impacta los resultados de muchas maneras, como se muestra a continuación.

GRÁFICO 13

COMPOSICIÓN SECTORIAL DEL ÚLTIMO QUINDENIO

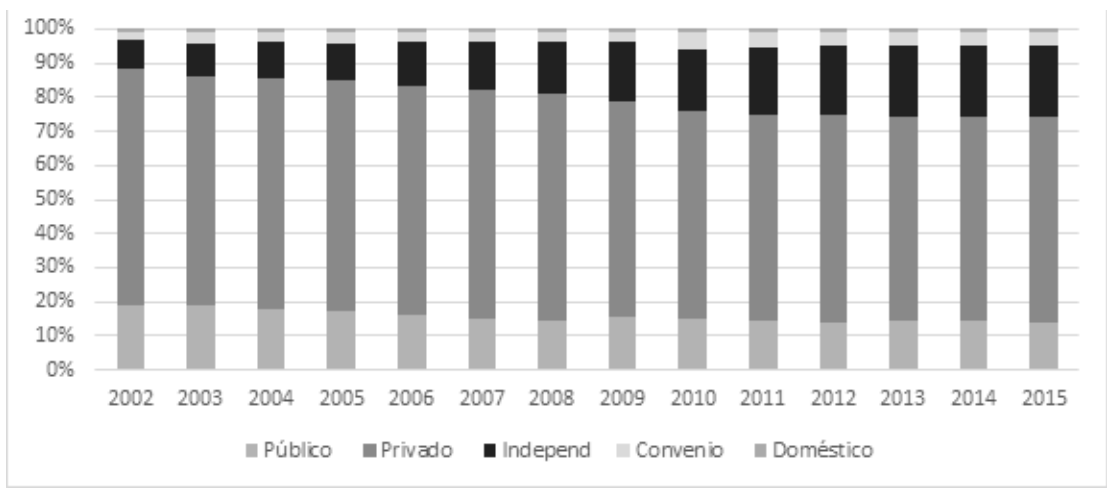

Fuente: Elaboración propia a partir de los datos presentados en el informe IVM-UCR (Arias et al., 2016).

Pero antes de pasar a cuantificar los posibles efectos de asumir una distribución constante dentro de la masa de cotizantes, es necesario analizar un poco más la situación actual del IVM. En el Cuadro No. 38 del informe IVM-UCR (Arias et al., 2016, p. 76) se muestra claramente los niveles 
de morosidad entre los sectores, dejando claro que son los "independiente", "convenio", y "doméstico" donde se podría mejorar el cobro, cambiando la verdadera representatividad entre sectores.

En resumen, existen dos factores que afectan la composición sectorial dentro de los cotizantes del IVM: oportunidades de aumento en cobertura, $y$ capacidad de mejorar los cobros de las cotizaciones. Dado que, como se mostró anteriormente, el crecimiento en cobertura y la reducción en morosidad no es uniforme entre sectores, entonces, el asumir que dicha distribución sectorial es constante se consolida en un nuevo sesgo dentro del estudio. Por ejemplo, cuando se define el salario promedio dentro del modelo del informe IVM-UCR (Arias et al., 2016, p. 244-245) se parte de la escala salarial del 2015 (por sexo, edad y años cotizados), pero no se considera el sector, asumiendo indirectamente que la representatividad sectorial es constante, $y$, por ende, el salario promedio no se vería afectado en el futuro, más que por inflación y aumentos salariales reales.

Primero que todo, hay que tomar en cuenta que los autores de éste artículo académico no contamos con la base de cuentas individuales, como sí lo tuvieron los participantes del equipo de la Escuela de Matemática de la UCR a la hora de elaborar el informe IVM-UCR (Arias et al., 2016), por lo que no fue posible hacer un cálculo de salarios promedio por edad, sexo, número de cotizaciones y sector, como se desearía. Dicho esto, se emplea un cálculo "proxy", que, a pesar de ser agregado, ejemplifica claramente el impacto. A partir de los cuadros No. 138 y No. 139 del informe IVM-UCR (Arias et al., 2016, p. 228-229) se puede tomar el salario promedio por sector y sexo para el año 2015, $y$ del cuadro No. 18 del informe IVM-UCR (Arias et al., 2016, p. 58) se deducen los porcentajes históricos de participación dentro de la población de cotizantes de las mujeres y los hombres, para cada uno de los sectores anteriormente mencionados.

Utilizando esta información, se puede calcular el salario promedio (ponderado) global (ambos sexos y todos los sectores) para cada uno de los porcentajes de representatividad históricos de la tabla, manteniendo los salarios sectoriales del 2015, de modo que se aísla el efecto en el salario promedio por causa de cambios en la composición sectorial. Se nota entonces que el salario promedio con la distribución del 2002 tiene un valor de 539.571 colones, mientras que el salario promedio con la composición del 2015 sería de 482.835 colones, es decir, se redujo en 56.736 colones, solamente por efecto de cambio en la composición sectorial. El gráfico 14 presenta el salario promedio para cada año.

GRÁFICO 14

COMPOSICIÓN SECTORIAL DE LA ÚLTIMA DÉCADA

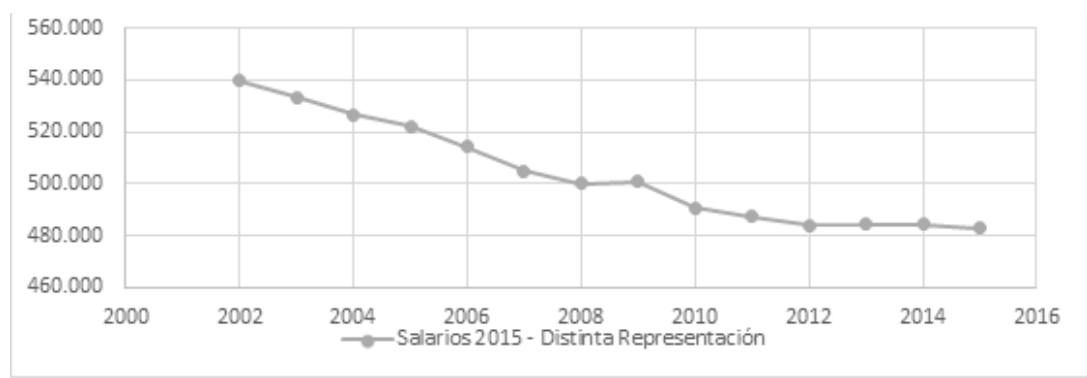

Fuente: Elaboración propia a partir de los datos presentados en el informe IVM-UCR (Arias et al., 2016).

Inicialmente no pareciera ser una diferencia importante, pero se debe recordar que este es el salario promedio para cada cotizante, de modo que al calcular la diferencia entre las masas salariales se logra dimensionar el efecto. Para ello, se toma la población de cotizantes generada por el modelo implementado por el equipo de la Escuela de Matemática, intentando medir el impacto (en colones del 2015) por efecto de diferencias en la distribución sectorial y la cantidad de cotizantes de cada año.

Con los cotizantes del 2015 se presenta una diferencia 1.034 .947 millones de colones, lo cual no es despreciable pues representa el $11,75 \%$ de la masa salarial del 2015 con la distribución del 
2015. Es decir, si el estudio se hubiera realizado en el 2002, y bajo el supuesto (como en este estudio) de que la distribución sectorial era constante, entonces se hubiera trabajado con una masa salarial inflada por un $11,75 \%$. La diferencia se incrementa conforme aumentan los cotizantes, al punto que para el año 2115 la diferencia es de 1.615 .030 millones de colones (del 2015).

El impacto en los ingresos del IVM, tomando en cuenta las primas de cotización vigentes del cuadro No. 128 del informe IVM-UCR (Arias et al., 2016, p. 196), se presentan el gráfico 15. Ahora queda más claro el impacto en las finanzas del IVM, puesto que para el 2015, sólo por efecto de cambio en la composición sectorial, los ingresos pasan de ser 836.620 millones de colones (composición sectorial del 2002) a ser 748.650 millones de colones (distribución sectorial del 2015), una diferencia de 87.970 millones de colones. En otras palabras, si el IVM experimentara un cambio en la composición sectorial similar, los ingresos proyectados estarían siendo sobreestimados por casi cien mil millones de colones.

\section{GRÁFICO 15 \\ INGRESOS DEL IVM CON DISTINTOS SALARIOS PROMEDIO}

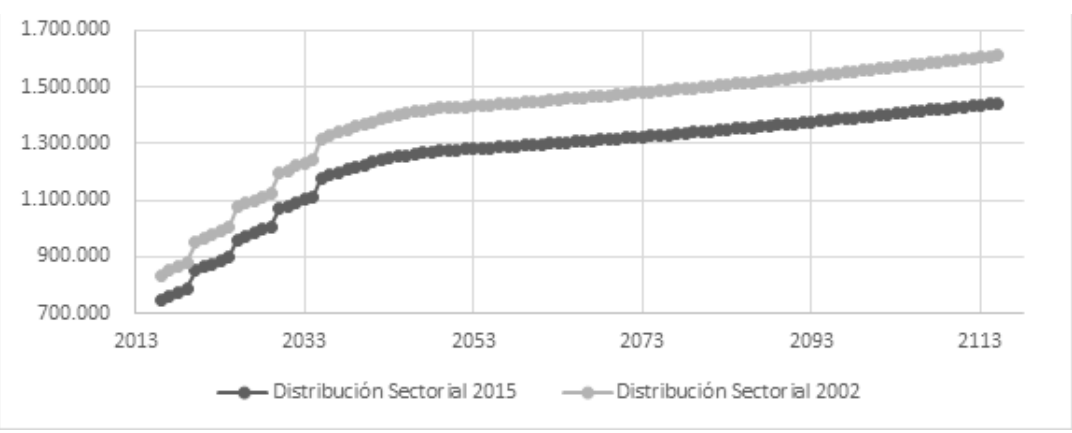

Fuente: Elaboración propia a partir de los datos presentados en el informe IVM-UCR (Arias et al., 2016).

Para poner este monto en perspectiva, se debe notar que el déficit entre los ingresos por aportes y el artículo 78 de la Ley de Protección al Trabajador, en el 2015, fue de 40.277 millones de colones. Es decir, si hoy el IVM tuviera una composición sectorial como la del 2002, no se necesitaría utilizar los intereses de las inversiones de la reserva para hacerle frente a sus obligaciones. Pero todavía hay más, hay que recordar que con más cotizantes la diferencia aumenta, de modo que para el 2115 la diferencia entre ingresos por cambios en la composición sectorial (2002 vs 2015) fue de 169.578 millones de colones (del 2015), más de 4 veces el déficit del 2015.

Se podría argumentar que el incremento de esta década fue atípico, producto de la Ley de Protección al Trabajador o por el artículo 7 de la Ley General de Migración y Extranjería No. 8764 (1ro de setiembre de 2009), pero que ya se estabilizó y que no se esperan cambios en este sentido. Sin embargo, este argumento sería falaz, como se desprende de los esfuerzos de la CCSS por aumentar la cobertura del sector doméstico. Por ejemplo, en la Gaceta del 21 de julio del 2017 salió publicado el "Reglamento para la Inscripción de Patronos y el Aseguramiento Contributivo de las Trabajadoras Domésticas" (La Gaceta, 2017, p. 23), el cual permite cotizar sobre una Base Mínima Contributiva reducida. De acuerdo a las estimaciones de la CCSS, para el 2021 se estaría aumentando la cobertura en el sector doméstico hasta un $40 \%$ o un $50 \%$, mientras que hoy representa solo un $11 \%$.

Esto tiene dos efectos sobre los ingresos: por un lado, aumenta la cobertura del sector doméstico cambiando la composición, $y$, por otro lado, el monto cotizado por cada patrón y cada empleado doméstico sería inferior. Ambos efectos van en la dirección de reducir el salario promedio. Asumiendo que se cumple la expectativa de la cobertura del 40\% de la PEA con esta política, la composición sectorial de las mujeres cambiaría (ver gráfico 16). 


\section{GRÁFICO 16 \\ COMPOSICIÓN SECTORIAL DE LAS MUJERES COTIZANTES}

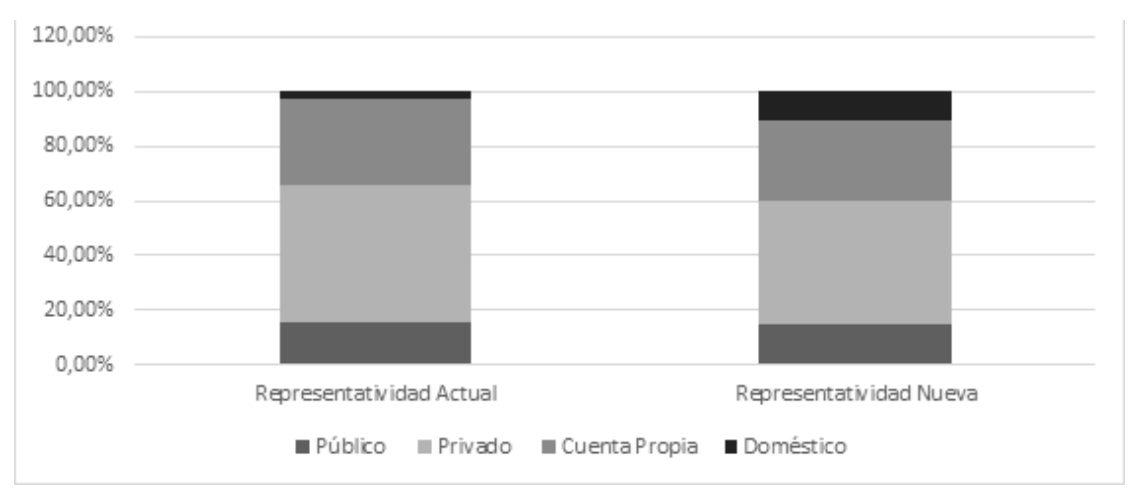

Fuente: Elaboración propia a partir de los datos presentados en el informe IVM-UCR (Arias et al., 2016).

Así, el sector doméstico llegaría a representar un 10,28\% de los cotizantes del IVM, prácticamente alcanzando al nivel del sector público que ronda el 14,41\%. Dado que el salario doméstico es el más bajo, esto provocaría que el salario promedio de las mujeres pase de 484 mil colones a 422 mil colones (con datos del 2015). Como ya se demostró, esta diferencia tiene un impacto importante dentro de los ingresos del IVM.

Del análisis retrospectivo realizado por el equipo de la Escuela de Matemática se desprende que la composición no es constante, y debió haberse tomado en cuenta, paralelamente con el aumento en cobertura, a aquellos sectores propensos a aumentar los cotizantes, y estimar de esta manera la nueva composición sectorial, así como el impacto en el salario promedio. Sin embargo, lo más recomendable sería trabajar con datos segregados sectorialmente, de modo que el impacto en el salario, producto de sus distintas tasas de crecimiento real, sea considerado dentro del estudio.

\section{Salario promediado vs. Salario esperado}

En los cálculos realizados por el equipo de la Escuela de Matemática se consideró la utilización de un salario de referencia (Arias et al., 2016, p. 245) que es el promedio representado por la fórmula:

$$
S P_{x, t, n}=\frac{1}{N u m} \sum_{k=0}^{N u m-1}\left(\frac{I P C_{n}}{I P C_{n-k}}\right) S_{x-k, t-k, n-k}
$$

donde "Num" depende del número de cotizaciones a considerar, el $\mathrm{IPC}_{\mathrm{n}}$ es el índice de precios al consumidor, $y S_{x, t, n}$ es el salario, todo esto para una edad "x", " $t$ " años cotizados, y en el año "n". Si se revisa el reglamento del IVM (CCSS, 2017) se encuentra que: "La pensión por vejez o invalidez se calculará con base en el promedio de los últimos 240 salarios o ingresos mensuales, devengados y cotizados por el asegurado, actualizados por inflación, tomando como base el índice de precios al consumidor." (art. 23).

Como se desprende de la fórmula (13), el promedio se realiza de manera continua sobre los 20 últimos años, asumiendo que el trabajador nunca dejó de laborar en ese periodo. En el programa 
macroeconómico del Banco Central (BCCR 2016) se presentan las probabilidades de transición entre "ocupado", "desempleado", e "inactivo". Ahí se nota que un ocupado tiene una probabilidad del 11\% de dejar de laborar, ya sea porque quedó desempleado o porque decidió dejar de trabajar del todo. Además, existe un $35,7 \%$ de probabilidad de que un desempleado consiga trabajo, mientras que ésta probabilidad es del 10,5\% para los inactivos. Esto nos indica que en realidad hay una cierta rotación entre ocupados $y$ desocupados, que influye en la continuidad con que ellos cotizan para el IVM.

La hipótesis de continuidad laboral implícita dentro de la fórmula (13) provoca que el salario de referencia, utilizado para el cálculo de las pensiones, sea más alto. Hay que notar que el salario Sx,t,n contiene un factor de aumento real "f" según el informe IVM-UCR (Arias et al., 2016, p. 245), el cual provoca que los últimos 240 salarios sean mayores en términos reales que cualquier otro salario anterior. Si una persona trabaja durante la etapa joven de su vida (15-39 años), para cuando cumple 40 años y deja de trabajar, ya habrá alcanzado las 300 cuotas necesarias para su jubilación, de modo que en el 2070 estará en posición de pensionarse (con 65 años), pero su salario de referencia no será ni cercano al obtenido con los salarios que podría haber recibido entre los 40 y 64 años.

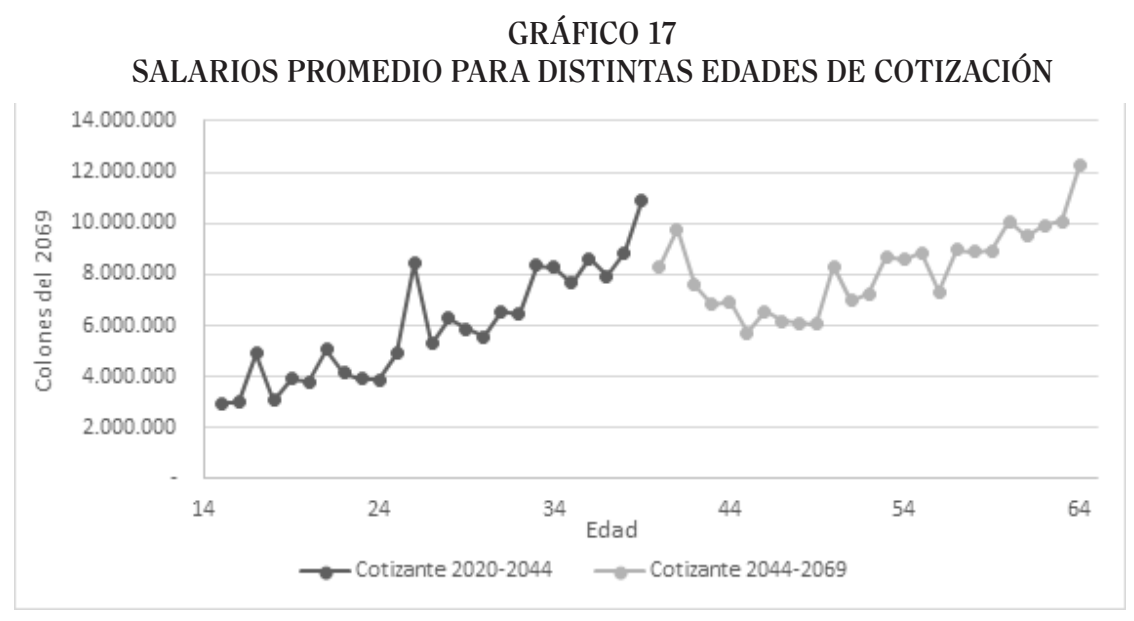

Fuente: Elaboración propia a partir de los datos de salario promedio utilizado en el informe IVM-UCR (Arias et al., 2016).

El gráfico 17 presenta los salarios con los que cotizaría si lo hace en los años de su juventud (15-39 años), o en los últimos años laborales (40-64 años). Si se utilizan los datos generados por el modelo de la UCR, $y$ la base de salarios promedio, para cada edad en cada año, utilizada para el informe IVM-UCR (Arias et al., 2016), se puede ver que el salario promedio para una persona que cumplió 65 años en el 2070, con 25 años de cotizaciones, sería el siguiente:

- Cotizó de 15 a 39 años: el afiliado inició sus cotizaciones en el año 2020 y entregó la última cotización en el 2044, dejando de cotizar en el 2045, año en que cumplió los 40 años. Este afiliado no vuelve a trabajar, y en el año 2070 cumple los 65 años, acogiéndose a la pensión de jubilación con el salario de referencia de los años cotizados. Al calcular, con el IPC respectivo, el promedio de los salarios de personas con edad $15+$ i para el año 2020 $+\mathrm{i}$, con $\mathrm{i}=0, \ldots, 24$, se tendría un salario de referencia igual a 6.535 .568 colones (del 2069). Para dimensionar este monto, deflatado a colones del 2015 , el salario de referencia sería de 646.200 colones.

- Cotizó de 40 a 64 años: el afiliado cotizó de manera continua desde el año 2045 (fecha de la primera cotización) al año 2069, dejando de cotizar en el año 2070, momento en que cumple los 65 años y se jubila, de modo que por reglamento el salario de referencia para el cálculo de la pensión corresponderá al promedio (ajustado por IPC) de los últimos 20 años de cotizaciones (del 2045 al 2069). Repitiendo el ejercicio del punto anterior, pero 
para este escenario, se obtiene un salario de referencia igual a 8.253.873 colones (del 2069), el cual deflatado a colones del 2015, sería de 816.096 colones.

A partir de estos cálculos se pueden obtener los respectivos montos de pensiones, siendo de 380.004 colones (del 2015) la pensión asociada al primer caso, y de 479.913 colones (del 2015) para el segundo, presentando una diferencia de casi 100.000 colones (del 2015) entre pensiones. Ya quedó claro como diferencias de este tipo pueden tener un impacto importante a la hora de agregar a todos los trabajadores/pensionados.

De esta forma, el valor de pensión presentado por el equipo de la Escuela de Matemática está sesgado, pues asume el peor caso posible, en el que todas las cotizaciones suceden con los salarios más altos. Al caso de un trabajador como el del ejemplo anterior, el informe IVM-UCR (Arias et al., 2016) le asigna un valor inflado por 100.000 colones, y para todos aquellos trabajadores que no realizan de manera continua sus últimas 240 cotizaciones, también se les estaría inflando erróneamente el monto de su pensión, elevando artificalmente los costos del IVM.

Aún más, si se hubiera realizado un estudio considerando la composición sectorial, es decir, segregado por sectores, entonces ésta diferencia sería aún mayor. Se debe notar que el monto de pensión varía producto de la diferencia salarial entre sectores (y sus tasas de crecimiento reales), de modo que, al considerar las cotizaciones realizadas en los distintos trabajos, se presentaría una discrepancia entre lo obtenido por el informe IVM-UCR de la Escuela de Matemáticas (Arias et al., 2016), y la realidad.

Se puede afirmar entonces, que la situación presentada en el informe de IVM-UCR (Arias et al., 2016) es pesimista, e infla los costos al sobreestimar los montos de pensión. Sin embargo, queda como una incógnita el impacto real de esta sobreestimación pues se carece de los datos para corroborarlo. En todo caso, lo más conveniente sería utilizar un salario de referencia esperado, donde se incluya la probabilidad de haber cotizado en cada año anterior. Para ello se puede utilizar las probabilidades de ser cotizante $\left(\mathrm{P}_{\mathrm{x}}, \mathrm{t}\right)$ y las densidades de cotización $\left(\mathrm{D}_{\mathrm{x}}, \mathrm{t}\right)$, para calcular este salario de referencia esperado, siendo un mejor estimador del salario de referencia real.

\section{Resultados esperados sin considerar varianza}

Es ampliamente conocido que el valor esperado (primer momento probabilístico) no presenta información completa respecto a una variable aleatoria en general. De ahí la conocida frase de que "es posible ahogarse en una piscina que tiene $10 \mathrm{~cm}$ de profundidad en promedio". Es por ello que es importante calcular otro tipo de información que complemente al valor esperado, tales como "la varianza" (segundo momento centrado), o un análisis de la cola de la distribución.

A lo largo del informe IVM-UCR (Arias et al., 2016) se presenta el escenario esperado para cada tripleta de hipótesis (cobertura, crecimiento real de los salarios, y rendimiento real de las inversiones). Sin embargo, los escenarios bajo y alto son irreales, debido a que suponen que se presentará el peor/mejor escenario supuesto en todos y cada uno de los años, lo cual es inverosímil (tiene una probabilidad de ocurrencia insignificante, técnicamente hablando). Bajo este entendido, no se recomienda utilizar escenarios de este tipo, sino que se promueve el uso de métodos estocásticos, tales como simulación con el método de Montecarlo, para capturar de mejor manera las posibilidades reales futuras.

Por ejemplo, es normal que en un año se presentara un rendimiento real de las inversiones igual a un 4\%, y que al año siguiente se observe que dicho rendimiento fue de 5,2\%, mientras que un año más tarde se experimente un rendimiento de 2,9\%, alternando entre distintos valores posibles. Sin embargo, en el informe IVM-UCR (Arias et al., 2016) se asume (para el escenario bajo) que todos los años el rendimiento sería del 2,9\%, algo casi imposible dado que el valor esperado es del $4 \%$. 
En finanzas se utilizan herramientas como árboles binomiales ${ }^{17}$ (en este caso serían trinomiales), para modelar este tipo de variables, asignando probabilidades a cada uno de los valores "posibles" (4\%; 5,2\%; 2,9\%), por lo que cada "nodo" del árbol tiene implícitamente una probabilidad asociada, y se pueden estimar valores críticos como el peor/mejor escenario posible (con cierto nivel de confianza), aprovechando que se conoce la probabilidad asociada a cada uno (nivel de significancia), $y$ apalancándose de conceptos como "Tail Conditional Expectation (TCE)", mismo que es ampliamente utilizado en finanzas bajo el concepto de "C-VaR" (ver Hull, 2006, p. 443-462). También es posible utilizar modelos continuos como el Vasicek o el Hull\&White (Brigo \& Mercurio, 2001).

Para clarificar este punto, si se supone que existe una probabilidad "p" de que el rendimiento real de las inversiones sea del 2,9\%. Bajo la metodología de árboles binomiales, la probabilidad de que se presente un escenario donde el rendimiento observado sea de 2,9\%, para los 100 años siguientes, sería de $\mathrm{p}_{100}$. Para poner esto en perspectiva, si se supone que la probabilidad "p" de observar un rendimiento real de las inversiones del 2,9\% en un año es del 95\%, entonces la probabilidad de que ocurra este rendimiento en 100 años consecutivos, sería de 0,6\% (menos del 1\%). Claramente es imposible que la probabilidad p ronde niveles del orden del 95\%, ya que el 2,9\% de rendimiento corresponde al percentil 35, según IVM-UCR (Arias et al., 2016, p. 294).

En caso de que el lector desee ver la teoría estocástica aquí mencionada, aplicada a estudios actuariales, puede remitirse al documento de Víquez, Víquez, Loría, Campos, y Mendoza (2018), titulado "Modelación de Poblaciones Vía Cadenas de Markov Tridimensionales". En este artículo los autores desarrollan un modelo para proyectar la población de una institución pública, y hacen uso de la Simulación de Montecarlo para generar escenarios y calcular el Valor en Riesgo Condicional (C-VaR) de los balances actuariales. En la sección 4 se desarrolla el algoritmo para la implementación del Montecarlo, el cual puede ser de utilidad para ejemplificar esta metodología de valores extremos (TCE).

\section{CONCLUSIONES}

El equipo de la Escuela de Matemática incumplió con los principios actuariales internacionales que obligan a revisar los datos (PEA-CCSS), validar el modelo y sus proyecciones (distribución de las nuevas generaciones, densidad de cotización, y afiliados proyectados), y supervisar/justificar algunos supuestos utilizados (composición sectorial uniforme, salario de referencia bajo hipótesis de cotización continua, entre otros). Como consecuencia, las observaciones aquí presentadas demuestran que los valores generados por el estudio IVM-UCR (Arias et al., 2016) del equipo de la Escuela de Matemática están sesgados y, por ende, las conclusiones derivadas del mismo no se sustentan sobre bases técnicas sólidas.

En vista de que hay que considerar el tiempo de implementación de las posibles reformas, es de primordial importancia conocer los momentos críticos de la forma más certera posible. Es por esto que se recomienda que se realicen las siguientes acciones a la hora de rehacer el estudio:

- Población económicamente activa corregida: es necesario rehacer todas las proyecciones de cotizantes y de recaudación del IVM sobre una base demográfica (Proyección de PEA) sin los sesgos anotados en este artículo. Se puede utilizar la PEA empleada en este artículo (base poblacional INEC-CCP con los respectivos supuestos sobre las tasas de actividad), con la cual se puede obtener una base de cotizantes más apegada a la realidad, corrigiendo así la subestimación de los ingresos encontrada.

- Densidad de cotización y probabilidad de cotizar: se debe corregir el error probabilístico de considerar la densidad de cotización de los afiliados como la probabilidad de ser cotizante, y agregar dentro del cálculo de los cotizantes sobrevivientes, el término faltante

17 El lector interesado en ver aplicaciones de árboles binomiales aplicado a rendimientos se puede dirigir a las referencias bibliográficas Björk (2004, p. 5-35), Cairns (2004, p. 29-48), van der Hoek y Elliott (2006). 
en la ecuación (7), de modo que el valor estimado no presente sesgos y no se sobreestime el tamaño de las nuevas generaciones. Para ello es necesario contar con la probabilidad de cotización correctamente estimada.

- Distribución de nuevas generaciones: no es correcto utilizar una distribución de nuevas generaciones agregada, como se mostró en este artículo. Se recomienda en su lugar, utilizar la PEA (INEC-CCP) por edades, y estimar a los cotizantes sobrevivientes para cada edad, de modo que se cuantifique la cantidad de "nuevas generaciones" por edad, sin utilizar agregados. Además, se recuerda que el modelo escogido debe respetar las relaciones intergeneracionales expresadas en la ecuación (12).

- Distribución sectorial: se recomienda trabajar cada sector por aparte, especialmente por el efecto que la composición sectorial tiene dentro de los ingresos del IVM. Si es posible se deben considerar los efectos de la nueva ley para trabajadoras domésticas, y continuar los análisis y/o proyecciones para cada sector, respetando sus relaciones y transiciones entre ellos, así como sus atipicidades (crecimiento real de los salarios, por ejemplo).

- Revisión de las proyecciones: se recomienda cumplir con el deber actuarial de validar el modelo, para lo cual se puede realizar un Back-Testing, o un método estadístico similar, de modo que se verifique que los resultados arrojados por el modelo son veraces, $y$ asegurarse así que el modelo es adecuado para utilizarse en el estudio actuarial del Régimen de Invalidez, Vejez y Muerte de la CCSS.

- Salario esperado: es recomendable trabajar con el salario esperado en lugar del salario promedio, especialmente si se están considerando las probabilidades de cotizar y las densidades de cotización para calcular los ingresos. Se sugiere emplear esta técnica probabilística para estimar el salario de referencia esperado, y así evitar sobreestimar los costos al utilizar la pensión más alta posible sin considerar su probabilidad de ocurrencia.

- Simulaciones y varianza/C-VaR: hay que notar que la población se genera con probabilidades que la convierten en un vector aleatorio multinomial, siendo el valor esperado de dicho vector el presentado en el informe IVM-UCR (Arias et al., 2016). Se sugiere utilizar técnicas como la "Simulación de Montecarlo", tal como el presentado en Víquez et al. (2018), o cálculos explícitos de varianza (técnicas más tradicionales - ver Bowers et al., 1997), para estimar la holgura del balance financiero-actuarial del IVM, en lugar de utilizar escenarios pesimistas/optimistas que poseen probabilidad cuasi-nula de ocurrencia.

Se espera que este artículo sirva para plantear la necesidad de rehacer este estudio, recalculando los pensionados, afiliados, cotizantes, entre otros, tomando en cuenta las observaciones aquí presentadas, de manera que se obtengan fechas más confiables para el momento en que se deberá hacer uso de la reserva, y el tiempo en que ésta se acabará. A partir de esos cálculos corregidos, se podrán conocer las acciones a realizar, sabiendo, con mayor certeza estadística, el tiempo con que se cuenta antes de arribar al tercer momento crítico. De este modo, se logrará contar con la información veraz y trascendental, sobre la cual se podrán tomar acciones en pro de la salud financiera del IVM. Además. teniendo un modelo validado, y aceptado por todas las partes, se podrá facilitar el diálogo entre ellas, favoreciendo los acuerdos a tomar por parte de los principales actores, además de volver más transparente los resultados obtenidos con el nuevo modelo.

\section{AGRADECIMIENTOS}

Se agradece al Centro Centroamericano de Población, en especial al Dr. Luis Rosero Bixby, por su enorme colaboración durante la elaboración de la PEA, así como grandes aportes a la hora del análisis demográfico. 
Igualmente, se extiende un agradecimiento a la Superintendencia de Pensiones y a la CCSS, por brindar los datos utilizados por los académicos de la Universidad de Costa Rica, permitiendo la confección de este artículo académico que es a su vez una revisión técnica del estudio actuarial realizado por la Escuela de Matemáticas para dicha institución.

\section{REFERENCIAS}

Actuarial Standards Board (junio, 2013). Exposure Draft. Modeling. American Academy of Actuaries. Recuperado de http://www.actuarialstandardsboard.org/asops/modeling-secondexposure-draft/

Arias, R., Barboza, L., \& Ramírez, J. (2016). Estudio actuarial del Seguro de Invalidez, Vejez y Muerte, administrado por la Caja Costarricense de Seguro Social. San José, Costa Rica: Escuela de Matemáticas, Universidad de Costa Rica.

Banco Central de Costa Rica (2016). Programa Macroeconómico 2016-2017. Recuperado de https:// www.bccr.fi.cr/seccion-publicaciones/publicaciones/programas-macroecon\%C3\%B3micos

Björk, T. (2004). Arbitrage Theory In Continuous Time. (2 ed.). Oxford University Press.

Bowers N., Gerber H., Hickman J., Jones D., \& Nesbitt C. (1997). Actuarial Mathematics, (2 ed.). Schaumburg: The Society of Actuaries.

Bringo, D., \& Mercurio, F. (2001). Interest Rate Models - Theory and Practice. https://doi. org/10.1007/978-3-540-34604-3

Cairns. A. (2004). Interest Rate Models - An Introduction. Princeton: Princeton University Press.

Centro Latinoamericano y Caribeño de Demografía (2016). Estimaciones y proyecciones de población total, urbana y rural, y económicamente activa. [Base de datos, Costa Rica, Estimaciones y Proyecciones de Población a Largo Plazo. 1950-2100. Revisión 2016]. División de Población de la CEPAL. Recuperada de https:/www.cepal.org/es/temas/proyeccionesdemograficas/estimaciones-proyecciones-poblacion-total-urbana-rural-economicamenteactiva

Centro Latinoamericano y Caribeño de Demografía (2017). Estimaciones y proyecciones de población total, urbana y rural, y económicamente activa [Base de datos, Costa Rica, Estimaciones y Proyecciones de Población a Largo Plazo. 1950-2100. Revisión 2017]. División de Población de la CEPAL. Recuperada de https:/www.cepal.org/es/temas/proyeccionesdemograficas/estimaciones-proyecciones-poblacion-total-urbana-rural-economicamenteactiva

Dirección Actuarial y Económica (2014). Costa Rica: La Población Económicamente Activa 19902013 y la Estimación de PEA para el Período 2014-2100. Manuscrito inédito.

Dirección Actuarial y Económica (2015). Valuación actuarial de largo plazo del IVM (con corte al 31 de diciembre de 2014). CCSS, Costa Rica. 
van der Hoek, J., \& Elliott, R. J. (2006). Binomial Models in Finance. https://doi.org/10.1007/0-387$31607-8$

Financial Reporting Council (2010). Technical Actuarial Standard M: Modelling. Reino Unido: FRC.

Caja Costarricense de Seguro Social (2017). Reglamento del Seguro de Invalidez, Vejez y Muerte. Caja Costarricense de Seguro Social, Costa Rica.

Hull, J. (2006). Options, Futures and other Derivatives. (6 $6^{\circ}$ ed.). England: Pearson Prentice Hall.

Instituto Nacional de Estadística y Censos - Centro Centroamericano de Población (2013). Estimaciones y Proyecciones de Población por Sexo y Edad 1950 - 2050. INEC-CCP, Costa Rica.

International Actuarial Association. (2012). International Standard of Actuarial Practice 1 (ISAP 1). Ottawa, Canada: International Actuarial Association.

International Actuarial Association. (2016). International Standard of Actuarial Practice 1A (ISAP 1A). Ottawa, Canada: International Actuarial Association.

Melinsky, E. (2015). Informe Final: Contratación Externa de Servicios Profesionales por parte de la CCSS y la SUPEN, para generar una valuación actuarial al Régimen de Invalidez, Vejez y Muerte. (Licitación Pública Nº 2013LI-000001-05101, CCSS-SUPEN) SUPEN, CCSS, San José, Costa Rica.

Nathal Actuarios y Consultores (2008). Informe Final de la Valuación Actuarial Practicada al Régimen de Invalidez, Vejez y Muerte de la Caja Costarricense del Seguro Social. (Licitación Abreviada 2007LA-000012-ODM, SUPEN) SUPEN, CCSS, San José, Costa Rica.

Organización Internacional del Trabajo (2010). Reporte sobre la validación de la evaluación actuarial del régimen de invalidez, vejez y muerte efectuada por la Caja Costarricense de Seguro Social. Ginebra: OIT.

Organización Internacional del Trabajo (2017). Consideraciones sobre el "Estudio actuarial del Seguro de Invalidez, Vejez y Muerte administrado por la Caja Costarricense de Seguro Social (con corte al 31 de diciembre del 2015)" elaborado por la Escuela de Matemática de la Universidad de Costa Rica (UCR). Ginebra: OIT.

La Gaceta, San José, № 179, viernes 21 de julio de 2017, p. 24.

Subramaniam, I. (1999). Actuarial Mathematics of Social Security Pensions. United Kingdom: International Labour Organization.

Víquez, J. J., Víquez, J. A., Campos, A., Loría, J., \& Mendoza, L. A. (2018). Modelación de poblaciones vía cadenas de Markov tridimensionales. Revista de Matemática: Teoría y Aplicaciones, 25(2), 185. https://doi.org/10.15517/rmta.v25i2.33608

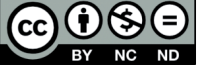

Todos los derechos reservados. Universidad de Costa Rica. Este artículo se encuentra licenciado con Creative Commons

Reconocimiento-NoComercial-SinObraDerivada 3.0 Costa Rica. Para mayor información escribir a revista.iice@ucr.ac.cr 